bioRxiv preprint doi: https://doi.org/10.1101/2022.02.19.481164; this version posted February 20, 2022. The copyright holder for this preprint (which was not certified by peer review) is the author/funder, who has granted bioRxiv a license to display the preprint in perpetuity. It is made available under aCC-BY-ND 4.0 International license.

\title{
Statistical Mechanical theory for spatio-temporal evolution of Intra-tumor heterogeneity in cancers: Analysis of Multiregion sequencing data
}

\author{
Sumit Sinha, ${ }^{1}$ Xin $\mathrm{Li}^{2}$ and D. Thirumalai ${ }^{2}, *$ \\ ${ }^{1}$ Department of Physics, University of Texas at Austin, Austin, TX 78712, USA. \\ ${ }^{2}$ Department of Chemistry, University of Texas at Austin, Austin, TX 78712, USA.
}

(Dated: February 20, 2022)

\begin{abstract}
Variations in characteristics from one region (sub-population) to another is commonly observed in complex systems, such as glasses and a collection of cells. Such variations are manifestations of heterogeneity, whose spatial and temporal behavior is hard to describe theoretically. In the context of cancer, intra-tumor heterogeneity (ITH), characterized by cells with genetic and phenotypic variability that co-exist within a single tumor, is often the cause of ineffective therapy and recurrence of cancer. Next-generation sequencing, obtained by sampling multiple regions of a single tumor (multi-region sequencing, M-Seq), has vividly demonstrated the pervasive nature of ITH, raising the need for a theory that accounts for evolution of tumor heterogeneity. Here, we develop a statistical mechanical theory to quantify ITH, using the Hamming distance, between genetic mutations in distinct regions within a single tumor. An analytic expression for ITH, expressed in terms of cell division probability $(\alpha)$ and mutation probability $(p)$, is validated using cellular-automaton type simulations. Application of the theory successfully captures ITH extracted from M-seq data in patients with exogenous cancers (melanoma and lung). The theory, based on punctuated evolution at the early stages of the tumor followed by neutral evolution, is accurate provided the spatial variation in the tumor mutation burden is not large. We show that there are substantial variations in ITH in distinct regions of a single solid tumor, which supports the notion that distinct subclones could co-exist. The simulations show that there are substantial variations in the sub-populations, with the ITH increasing as the distance between the regions increases. The analytical and simulation framework developed here could be used in the quantitative analyses of the experimental (M-Seq) data. More broadly, our theory is likely to be useful in analyzing dynamic heterogeneity in complex systems such as super-cooled liquids.
\end{abstract}

PACS numbers:

\section{INTRODUCTION}

Heterogeneity is pervasive on all scales in abiotic and biological systems. In glass forming systems, the dynamics in a seemingly homogeneous sample shows that slow and fast moving particles coexist within one sample, a phenomena referred to as dynamical heterogeneity [1, 2]. As a consequence, there are variations in properties (distribution of energies for example) in different regions within a single sample of a supercooled liquid near the glass transition [2, 3]. In biology, it is becoming increasingly clear that heterogeneity dominates on all length scales 4, from molecules to chromosome conformations, and in the behavior of different cells. Nowhere is the heterogeneous behavior more relevant than in cancers in which there are genotypic and phenotypic variations among different sub-populations within a single tumor, which is commonly referred to as intratumor heterogeneity (ITH) [5] 8 .

Cancer is an evolutionary disease that is, in most cases, triggered through accumulation of harmful mutations over a period of several decades [9]. Both exogenous (external environmental factors) and endogenous factors (such as DNA replication errors) [10, 11] give rise to harmful mutations. Thus, cancers may be classified as, "exogenous" (melanoma, lung etc.) and "endogenous" (kidney, brain etc.) based on the origin of mutations. With the advent of next-generation sequencing technologies, one can characterize the genetic information of cancer cells at unprecedented whole-genome level resolution [12]. A few hundreds of "driver mutations", which bestow significant growth or fitness advantage to the cancer cells, have been discovered in different cancers through cancer genome projects [13]. However, the evolutionary dynamics of cancers, which is encoded in the mutational history, is still elusive [14]. In particular, a quantitative description of ITH, which could be readily used to analyze experimental data, does not exist.

In the linear evolution model for tumor growth, proposed in a seminal work [15], cells accumulate driver mutations sequentially, which outcompete the preexisting clonal population through selective sweeps. However, most cancers exhibit extreme heterogeneity, as cells with distinct genetic and phenotypic characteristics, co-exist within a single

*Electronic address: dave.thirumalai@gmail.com* 
tumor 16 18. This finding is in apparent contrast to the linear evolution model. As an alternative, branched evolution in which multiple subclones grow simultaneously in the same tumor, with rare selective sweeps, is supported by many next-generation sequencing experiments [19-21]. Besides, neutral evolution, marked by lack of selection or fitness advantage, has been introduced for several cancer types 22 24. Recently, a punctuated evolution 25 model was invoked for some cancers. Instead of accumulating mutations sequentially and gradually, as in the previous models, a burst of mutations may occur in a short period of time in the early stage of tumor evolution. Although there are several models for cancer evolution [26, 27, it is universally agreed that intra-tumor heterogeneity (ITH) is common among different cancers. Pervasive ITH is one of the leading reasons for cancer resistance to conventional therapy[28]. Hence, the study of ITH is of utmost importance both as a fundamental problem in the physics of cancers and for cancer treatment.

Due to the spatial genetic variations among cancer cells, traditional single biopsies greatly underestimate the ITH in cancer patients [18, 20, 29. Lack of quantitative understanding of the spatio-temporal variation in ITH is often blamed for ineffective therapeutic strategies as they target only part of tumor cell populations [19, 20, 30]. Although known for decades, it is possibly only through Multiregion sequencing (M-Seq) data has it became abundantly clear that ITH is pervasive in many cancers. In M-Seq experiments, multiple regions in a single tumor are sequenced 19, 20. These experiments for several cancers and many patients reveal that there are substantial region-to-region diversity in the mutated genes. Therefore, any theory for ITH must take spatial variations into account [29, 31]. The implications of the M-Seq data analyses serves as the main inspiration for the present theoretical study.

Previously, several theoretical studies estimate ITH of cancer patients from sequencing data 31 40. However, these studies have limitations, preventing their use in estimating ITH from M-Seq experiments. Patient's ITH is often evaluated using the Simpson's index [32, 33] where a well-mixed (spatially homogeneous) tumor evolution model is considered, which is clearly not suitable for analyzing the M-Seq data. In a pioneering study Gonazalez-Garcia et. al. 31, which took into account spatial variations, depicted the in silico results as a two-dimensional map, like the one in Fig. 1. Because they investigated the frequency of cell populations with mutations in only two genes, it limits the applicability of the model to M-Seq data. In a promising study, a spatial computational tumor model was created [37, which showed that shared genetic alterations decrease as a function of inter-cellular distance. However, they [37] did not draw firm conclusions on ITH. In particular, they did not compare the available data from M-Seq experiments and the predictions from the computational model.

Our work is closest in spirit to the seminal studies by Curtis and coworkers, who used an Approximate Bayesian Computation methodology 23] to propose the Big Bang dynamics, first in the context of human colorectal cancer. They established that, after initially acquiring mutations, the tumor progresses without stringent selection. Subsequently, they [4] created a spatial tumor growth simulation model to predict the heterogeneity determined in the multi-region sequencing experiments. Although the initial studies focused on colorectal cancer, it was later realized that effective neutrality (tumor growth without strict selection) is likely to be more generally valid 24 for other cancers as well. Remarkable as these studies are, to the best of our knowledge, no prior study has developed a theoretical framework to calculate ITH analytically, so that it can be readily used to analyze M-Seq experiments. We fill this missing gap in this work by creating a statistical mechanical theory complemented by simulations.

To quantitatively estimate ITH revealed in M-Seq experiments, we develop a theory applicable to solid tumors based on neutral evolution of tumor in three dimensions inspired by recent studies [23, 24. We note parenthetically that although there is considerable debate on the nature of tumor evolution (neutral or with selection, linear and punctuated), it is universally accepted that ITH is pervasive in many cancers. Our theory has two parameters: (i) Cell division probability $(\alpha)$, and (ii) Mutation probability $(p)$. The central results of the study are: (I) A closed form expression for ITH is obtained, which is written as, $\operatorname{ITH}(\alpha, p, t)=G(p, t) F(\alpha)$, where $G(p, t)$ is the ITH with $\alpha=1$. The function $F(\alpha)$ is the scale factor that accounts for the possibility that $\alpha$ is less than unity $(0.5<\alpha<1)$. (II) $G(p, t)$ is determined by the correlations induced in cell lineages that arise due to cell division. The magnitude of $G(p, t)$ depends on the geometry in which the tissue is embedded. (III) The scale factor, $F(\alpha)$, increases linearly upon increasing $\alpha$, physically implying that ITH decreases on increasing apoptosis (i.e reducing cell division probability). (IV) The utility of our theory, quantified using Pearson correlation $(\rho)$, is established by directly comparing with the M-Seq data for cancers originating in four tissue types-Skin [42] (8 patients), Lung [43, 44] (60 patients), and Kidney 20] (10 patients). We obtained $\rho=0.97$ for skin, $\rho=0.87$ for lung and $\rho=0.51$ for Kidney, indicative that our theory can capture M-Seq experiments for exogenous cancers (i.e skin and lung). (V) We find that the effectiveness of our theory in capturing experiments is related to the spatial distribution of tumor mutation burden (TMB), quantified using the coefficient of TMB variation $\left(c_{v}\right)$. Exogenous cancers have smaller $c_{v}$ (similar to predictions from theory) compared to endogenous cancer and hence the theory captures the ITH observed cancers originating in skin and lung. The theory provides a testable framework for analyzing ITH extracted from M-Seq experiments. (VI) Besides confirming the validity of the theory, the simulations vividly illustrate ITH as manifestation of massive variations in the Hamming distance from one region to another. This picture, that emerges from the simulations, is in accord with the M-Seq experiments. 
bioRxiv preprint doi: https://doi.org/10.1101/2022.02.19.481164; this version posted February 20, 2022. The copyright holder for this preprint (which was not certified by peer review) is the author/funder, who has granted bioRxiv a license to display the preprint in perpetuity. It is made available under aCC-BY-ND 4.0 International license.

\section{EXTRACTION OF ITH FROM M-SEQ DATA}

\section{A. Analysis of Experimental data}

We first analyzed the experimental data because it sets the stage for the development of the theoretical model. We used the M-Seq data-sets that were collected for cancers in four tissue types -Skin [42], Lung [43, 44], Esophagus [45] and Kidney [20. M-Seq [19, 20, 42, 43, 45, 46] refers to whole exome sequencing of multiple regions in a cancerous tumor (Figure 17). A typical readout of a M-Seq data is represented as a matrix (see Figure 1b), where the nonsynonymous mutations detected across all the regions are displayed in the form of a binary heat map. The string (a one dimensional array that contains the list of genes) of length, $n$, in the M-Seq data is the total number of uniquely mutated genes in the whole tumor. In principle, the blueprint of cancer evolution over time scales spanning decades is contained in these readouts, 47. which is illustrated schematically in Figure 1]. Depending on the pattern of the heat-maps, a measure that quantifies ITH may be calculated for each readout. The average ITH values (explained below) are unique to each patient. We used Hamming distance (HD) as a measure of ITH.

We collected M-Seq readouts from four cancer types. Three of them (skin, lung and esophagus cancers) arise predominantly due to exogenous factors, while kidney cancer is caused by endogenous factors [10]. We analyzed eight, sixty, two and ten publically available patient data for skin [42], lung [43, 44], esophageal [45] and kidney [20] cancers, respectively.

ITH from experimental readouts: The M-Seq data, represented as a matrix whose elements represent the biopsied regions (columns in Figure 1b) and the sequenced genes (rows in Figure 1b), generate averages over the cells in a specific region. We use Figure $1 \mathrm{p}$ to illustrate how ITH is extracted from M-seq data. There are seven regions, labeled $\mathrm{R} 1, \mathrm{R} 2, \mathrm{R} 3, \mathrm{R} 4, \mathrm{R} 5, \mathrm{R} 6$, and $\mathrm{R} 7$. Let each column be $X^{R_{i}}$, where $i \in[1,2,3,4,5,6,7]$. The length, $n$, of each row $\left(\left|X^{R_{i}}\right|\right)$ gives the number of genes, which is 83 in Figure $1 \mathrm{~b}$. To quantify the average heterogeneity associated with the data in Figure 1 b, we calculated the Hamming distance (HD) [35, 48, 49], between all the pairs in the seven regions. The total number of distinct pairs, denoted by $M$, is $C_{2}^{M}=C_{2}^{7}=21$ in Figure 1 b. Hamming distance between columns belonging to two regions, $A$ and $B$, is given by, $H D\left(X^{R_{A}}, X^{R_{B}}\right)=\frac{1}{n} \sum_{i=1}^{n}\left|X_{i}^{R_{A}}-X_{i}^{R_{B}}\right|$, where $|.$.$| is the absolute value. Note that 0 \leq H D\left(X^{R_{A}}, X^{R_{B}}\right) \leq 1$ with 0 being homogeneous (no spatial variation), and $H D\left(X^{R_{A}}, X^{R_{B}}\right)=1$ implies that the tumor is maximally heterogeneous. Table 1 in the SI shows the Hamming distances between the 21 pairs. After calculating the HD among all the possible pairs, we determined the average value $\langle\mathrm{HD}>(0.17$ in Figure $1 \mathrm{p})$. Figure $1 \mathrm{c}$ shows the distribution of $H D$ between all the 21 pairs. The $H D$ values range from $\approx 0.02$ to $\approx 0.40$, with a mean of 0.17 . The data, as presented, already shows that there are variations at the mutation level between different regions in a single tumor. We adopt the same procedure to calculate the ITH, measured in terms of the HD, for each patient from the M-Seq readout for the other cancer types (see the SI figures S2-S6 for details).

\section{B. Theory for ITH with neutral evolution}

The theoretical model is based on generating evolutionary trajectories through space and time using 3D lattice representation of the tumor (illustrated in Figure S11 in the SI). Besides occupying a site on the lattice, each cell $i$ carries genetic information in the form of a string $X^{i}$ whose length, $n$, is assumed to be a constant. This is similar to the M-seq experiments (see Figure 1b with $n=83$ ). Each site in the string represents a gene in the DNA. A gene that is not mutated is denoted by 0 while a mutated gene is represented by 1 , which is the binary representation adopted in the M-seq experiments. We initialize the tumor evolution by placing a single cell with $X^{i}=0$ at the center of the $3 \mathrm{D}$ lattice. The tumor evolves and generates an evolutionary trajectory in space and time according to the following rules.

(i) Cell division and replication: At each time $t$, a cell could divide and give birth to a daughter cell, with probability, $\alpha$, provided one of the 26 neighboring sites in the 3D lattice is vacant. We are considering a growing tumor through punctuated evolution 25] initially, with a few driver mutations, which implies that $\alpha>0.5$. After cell division, the genetic information of the parent $(\mathrm{P})$ cell is copied to the daughter $\left(X^{D}=X^{P}\right)$. This biologically realistic copying mechanism is an essential part of the model as it leads to correlations in space and time [50, 51].

(ii) Mutations: During each time-step, a cell could acquire mutations at non-mutated sites with probability, $p$. Acquisition of mutation is independent of cell division, which is the case for exogenous mutations. For instance, let us denote the cell by index $m$, non-mutated site by $i$ and the string by $X_{i}^{m}$. The above statement can be represented mathematically as, $\mathbb{P}\left[X_{i}^{m}(t+1)=1 \mid X_{i}^{m}(t)=0\right]=p$. It follows that the probability that a site is not mutated at $t+1$, given that it was not mutated at time $t$, is $1-p$, which we write as $\mathbb{P}\left[X_{i}^{m}(t+1)=0 \mid X_{i}^{m}(t)=0\right]=1-p$. 
(iii) Mutations are neutral: The mutations do not add fitness or selective advantages to cells since we only consider neutral evolution [26] at later stages of tumor growth although initially we used punctuated evolutionary process. Preliminary results using evolution with selection that confers fitness advantage to cancer cells yields qualitatively similar results (see the Figure S12 in the Supplementary Information (SI) ).

(iv) Mutations are irreversible: We also assume that a site (gene in the M-Seq data), on string $X^{m}$, once mutated cannot revert to the original status in the future, as is assumed in other studies [52]. This can be rationalized by the following argument. In M-Seq experiments, the sites in the strings $X^{m}$ code for genes. Suppose we consider a gene, $\mathrm{Q}$, with length $L$ base-pairs (bp), then the ratio of reverse mutation to forward mutation probabilities is negligible. Assuming that only a single nucleotide in gene $\mathrm{Q}$ is mutated, the reverse mutation probability $\left(\mathcal{P}_{b}\right)$ is $\mu_{n}$, where $\mu_{n}$ is the mutation probability for a nucleotide. Note that mutation probability of a single nucleotide $\mu_{n}$ is different from mutation probability of gene, $p$. Similarly, the forward probability $\left(\mathcal{P}_{f}\right)$ is $(L-1) \mu_{n}$. Therefore, $\frac{\mathcal{P}_{b}}{\mathcal{P}_{f}}=\frac{1}{L-1} \rightarrow 0$, because the average gene length $L \approx 10-15 k b p[53$, 54]. Therefore, once a nucleotide in the gene is mutated, the probability of reversing the mutation at that specific nucleotide is negligible. Mathematically, the irreversibility of mutations is written as, $\mathbb{P}\left[X_{i}^{m}(t+1)=0 \mid X_{i}^{m}(t)=1\right]=0$.

(v) Apoptosis: At any time-step, a cell may undergo apoptosis with probability $1-\alpha$. Given the five rules, our goal is to calculate the average ITH, expressed as the mean HD, in a tumor containing $N$ cells. There are only two parameters in the model, cell division probability $(\alpha)$, and mutation probability $(p)$. Let $t$ be the time for the tumor to grow $N$ cells. We define the $\operatorname{ITH}(t)$ of the tumor as,

$$
I T H(t)=\frac{2}{N(N-1)} \sum_{i=1}^{N} \sum_{j=i+1}^{N} H D\left[X^{i}(t), X^{j}(t)\right]
$$

where $H D\left[X^{i}\left(t_{N}\right), X^{j}\left(t_{N}\right)\right]$ is the Hamming distance (HD) between the DNA strings in cells $i$ and $j$ at time $t$. The method used to compute HD theoretically is similar to the one we employed to analyze M-seq experiments (see SI Table 1).

\section{Stochastic simulations for tumor growth}

To validate the theoretical results, we developed a three-dimensional lattice model of tumor evolution (see the details in section III of the SI.) The cellular automaton based simulations mimic the theoretical rules for tumor evolution, which are described in the previous section. For all the simulation results, we used the string length, $n=300$, which is comparable to the string length in the M-Seq experiments.

\section{RESULTS}

\section{A. ITH under neutral evolution}

In terms of cell division probability $(\alpha)$ and mutation probability $(p)$, the $\operatorname{ITH}(\alpha, p, t)$ of a neutrally evolving tumor in three dimensions (3D) may be expressed as,

$$
\operatorname{ITH}(\alpha, p, t) \equiv G_{3 D}(p, t) F(\alpha)=2 x_{t}\left(1-x_{t}\right)(0.2 \alpha+0.77) .
$$

In Eq2 $G_{3 D}(p, t)=2 x_{t}\left(1-x_{t}\right)$ is the value of ITH when $\alpha=1, x_{t}=(1-p)^{t}$, and $F(\alpha)$ is a scale factor, which ensures that $\alpha$ is less than unity $(0.5<\alpha<1)$. This is the central result in this work. The seemingly simple analytic expression for $\operatorname{ITH}(\alpha, p, t)$ in Eq. 2 shows that it factorizes into a product of the two functions one of which depends solely on $p$ and the other monotonically increases with $\alpha$.

Branches, correlated and uncorrelated evolution: Let us first provide insights into Eq. 1 for tumor evolution with $\alpha=1$. Figure 2a illustrates a sample evolutionary tree at $t=0,1,2,3$ for $\alpha=1$. Figure S15 shows the snapshots of tumor evolution for $\alpha=1$ obtained from lattice simulations. In this case, the growth process may be understood in terms of a directed tree, composed of cells and directed edges. Because $\alpha=1$, once a cell or an edge is created it remains throughout the evolutionary process. The edges provide the child-parent relationship between two cells. For instance, in Figure 2 a, the edges from $1 \rightarrow 2$ means that cell 2 was born from cell 1 . The evolutionary tree, which is an imprint of the trajectories, has many branches. A branch is a unique path traversed from the origin (cell 1) to the last cell (no directed edge from a node) following the directed edges. In Figure 2 a, $1 \rightarrow 2 \rightarrow 3 \rightarrow 5$ is the longest branch at $t=3$. In $3 \mathrm{D}$, these branches are curved implying that the Euclidean distance from cell 1 to the end 
of the branch (the corresponding leaf node), is not equal to the number of nodes in the branch. However, because cell-division occurs only if there is a vacant neighbor, we assume that the curvature associated with the branches are negligible, rendering them as linear. With this assumption, a 3D evolutionary tree may be pictured as consisting of many linear branches. The linear branches correspond to the evolution of cells on a semi-infinite 1D lattice where growth occurs unidirectionally away from the origin. For this reason it is instructive to calculate ITH for a 1D-semi infinite lattice. For completeness, we also estimate the number of branches in all the geometries - 1D semi-infinite, 1D infinite, 2D and 3D evolutionary trees. Let the number of branches in an evolutionary tree be $N_{b r}$. For semi-infinite lattice $N_{b r}=1$, for infinite 1D lattice $N_{b r}=2$, for a 2D evolutionary tree $N_{b r} \sim R$, and for a 3D evolutionary tree $N_{b r} \sim R^{2}$. Here, $R$ is the radius of the tumor in two and three dimensions. In $d$ dimensions, $N_{b r} \sim R^{d-1}$.

The heterogeneity between the cell pairs depends on whether they belong to the same branch or not. Pairs of cells sharing a common branch will have correlations in their genetic information whereas cells that do not share any common branch segment will evolve independently. For instance, in Figure 2 a, the evolution of node 8 is independent of node 5 as they belong to different branches. On the other hand, the evolution of node 5 is correlated with evolution of node 2 because they are in the same branch. Figures $2 \mathrm{~b}$ and 2 represent the schematic for cells undergoing independent and correlated evolution. To evaluate $G_{3 D}(p, t)$, we first calculate heterogeneity among cells that undergo independent and correlated evolution.

Heterogeneity in independently evolving cells: Figure $2 \mathrm{~b}$ shows a schematic of cell pairs, labelled as 1 and 2 , evolving independently in time. The genes in their respective DNA, $X^{1}$ and $X^{2}$, are not mutated at time $t=0$ ( $X_{i}^{1}=X_{i}^{2}=0$ for $\left.1 \leq i \leq n\right)$. Given the rules for generating the evolution trajectories, presented in section II.B, we evaluate the average heterogeneity among a pair of cells evolving independently, $\left\langle H D_{\text {ind }}\left[X^{1}(t), X^{2}(t)\right]\right\rangle$. Here, $\langle\ldots\rangle$ represents the ensemble average over all the cell pairs (see SI for an additional explanation).

It is shown in the Appendix that the average heterogeneity between a pair of cells evolving independently $\left(\left\langle H D_{\text {ind }}\left[X^{1}(t), X^{2}(t)\right]\right\rangle\right)$ is,

$$
\left\langle H D_{\text {ind }}\left[X^{1}(t), X^{2}(t)\right]\right\rangle=2 x_{t}\left(1-x_{t}\right),
$$

where $x_{t}=(1-p)^{t}$ is the probability that a gene is not mutated till time $t$.

Heterogeneity in cells with correlated evolution: Figure 2 shows a schematic of an evolutionary trajectory for cell pairs, labelled as 1 and 2, that is correlated in time. During correlated evolution, cell pairs have a common ancestor till $t>0$. Let us consider the case when a cell evolves acquiring mutations from $t=0$ till time $t$. After time $t$, it gives birth to cell 2. During the birth process, the content in string $X^{1}$ is copied to string $X^{2}$, which introduces correlations between $X^{1}$ and $X^{2}$. We wish to evaluate $\left\langle H D_{\text {corr }}\left[X^{1}(t+s), X^{2}(t+s)\right]\right\rangle$ between pairs of cells that undergo correlated evolution at some later time $t+s$. The average heterogeneity $\left(\left\langle H D_{\text {corr }}\left[X^{1}(t), X^{2}(t)\right]\right\rangle\right)$ in this case is (see Appendix for details),

$$
\left\langle H D_{\text {corr }}\left[X^{1}(t+s), X^{2}(t+s)\right]\right\rangle=2 x_{t+s}\left(1-x_{s}\right) .
$$

The expression in Eq. 4, gives the average heterogeneity between two cells which undergo a common evolution till $t$ but subsequently evolve independently till $t+s$. If we set $t=0$ in Eq4 we obtain the result for independent evolution in Eq.3. The result in Eq. 4 is particularly interesting because it shows that the two cells are correlated in time because of the copying mechanism (daughter cell inherits all the genetic information in the mother cell) during cell-division. It has previously been noted that cell division induced correlations among cells share a common lineage [50, 51. Because of correlated evolution, cell pairs that undergo correlated evolution have smaller heterogeneity compared to cells pairs that evolve independently $\left(\frac{H D_{i n d}\left[X^{1}(t+s), X^{2}(t+s)\right]}{H D_{\text {corr }}\left[X^{1}(t+s), X^{2}(t+s)\right]}=\frac{1-x_{t+s}}{1-x_{s}}>1\right)$.

Role of Branches in an evolutionary tree: Having calculated the heterogeneity among cells evolving independently and in a correlated manner, we can evaluate the average heterogeneity within the 3D tumor at time $t$ for $\alpha=1, G_{3 D}(t, p)$. The expression for $G_{3 D}(t, p)$ is given as,

$$
G_{3 D}(t, p)=\frac{\int_{1}^{R} \int_{1}^{r_{1}} r_{1}^{2} r_{2}^{2} F_{3 D}\left(r_{1}, r_{2}, t\right) d r_{2} d r_{1}}{\int_{1}^{R} \int_{1}^{r_{1}} r_{1}^{2} r_{2}^{2} d r_{2} d r_{1}}
$$

where $R$ is the radius of the tumor, and $F_{3 D}\left(r_{1}, r_{2}, t\right)$ is the heterogeneity for a pair of cells at distances $r_{1}$ and $r_{2}$ from the origin at time $t$. Without loss of generality, we assume that $r_{1} \geq r_{2}$. The general expression of $F_{3 D}\left(r_{1}, r_{2}, t\right)$ may be written as,

$$
F_{3 D}\left(r_{1}, r_{2}, t\right)=P_{b r, 3 D}\left\{2 x_{t}\left(1-x_{t-r_{2}}\right)\right\}+\left(1-P_{b r, 3 D}\right)\left\{2 x_{t}\left(1-x_{t}\right)\right\}
$$


where $P_{b r, 3 D}$ denotes the probability that both the cells belong to the same branch of the 3D evolutionary tree. In 3D, $P_{b r, 3 D}=\frac{1}{\frac{4}{3} \pi r_{1}^{2}}$. Note that to obtain the expression of $P_{b r, 3 D}$, we assumed that the branches are linear. On evaluating the integrals in Eq. 5, we obtain an analytical expression for $G_{3 D}(p, t)$ given by,

$$
\begin{aligned}
& G_{3 D}(p, t)= \\
& \frac{3(1-p)^{t}}{\pi\left(R^{3}-1\right)^{2}}\left(-\frac{2}{3} \pi\left(R^{3}-1\right)^{2}\left[(1-p)^{t}-1\right]+\frac{3}{4}(R-1)^{2}(1-p)^{t}\left(R^{2}+2 R+3\right)\right. \\
& -\frac{(1-p)^{t-R-1}}{\log ^{4}(1-p)}\left[9(1-p)^{R}\{-6+\log (1-p)(2(R-3)+\log (1-p)\{2 R-3+(R-1) \log (1-p)\})\}\right. \\
& -9(p-1)[6+R \log (1-p)\{R \log (1-p)+4\}]])
\end{aligned}
$$

Simulations: We performed cellular automaton type simulations to validate the results given in Eq. 7 . Figure 2 f shows that the theoretical predictions $(\mathrm{Eq} .7)$ and the simulation results are in excellent agreement. Although the analytic result for $G_{3 D}(p, t)$ is complicated, it can be drastically simplified by noting that $P_{b r, 3 D} \sim 0$ in Eq. 6 . Therefore, the average heterogeneity $(\alpha=1)$ in $3 \mathrm{D}$ becomes $2 x_{t}\left(1-x_{t}\right)$, which means that the branches (deemed to be linear) evolve independently. The maximum heterogeneity in this case is $\approx 0.5$. Hence, we have a simple expression for $G_{3 D}(t, p)$, which can be written as,

$$
G_{3 D}(p, t) \approx G_{3 D}\left(x_{t}\right)=2 x_{t}\left(1-x_{t}\right)
$$

Since, $P_{b r}$ is specific to the geometry of the tissue, we explored the dependence of heterogeneity in different geometries (see Appendix for details).

Derivation of $F(\alpha)$ : Although Eqs. 7 and 8 could be obtained analytically, determination of $\alpha$-dependent $F(\alpha)$ (Eq. 2 requires simulations. The term, $F(\alpha)$ in Eq. 2, accounts for the effect of changing birth probability, $\alpha$. Figure 3 a shows ITH as a function of $x_{t}$ for different $\alpha$ values. The extent of ITH decreases as $\alpha$ decreases. Upon decreasing $\alpha$, the probability of a vacant site in the neighborhood of a cancer cell increases. As a result, the cancer cell gives birth to a daughter cell with similar genetic information. Therefore, the similarity in the genetic information between the neighboring cells increases, which decreases the overall heterogeneity of the tumor.

To extract the functional form of $F(\alpha)$, we fit $\frac{I T H\left(x_{t}=0.5\right)}{G_{3 D}\left(x_{t}=0.5\right)}$ versus $\alpha$ to a line, as shown in Figure $3 p$, which yields,

$$
F(\alpha)=(0.2 \alpha+0.77)
$$

Since, we obtained Eq. 9 by fitting $\frac{I T H\left(x_{t}=0.5\right)}{G_{3 D}\left(x_{t}=0.5\right)}$ at $x_{t}=0.5$, there is no guarantee that the linear dependence should hold for all values of $x_{t}$. In order to show that $F(\alpha)$ is accurately given by Eq. 9 for all values of $x_{t}$, we plotted $\frac{I T H}{F(\alpha)}$ in Figure 3. Figure 3c shows that all the curves, corresponding to different values of $\alpha$, collapse onto the same master curve, which coreesponds to $G_{3 D}(p, t)$. Note, that $F(\alpha) \approx 1$ when $\alpha=1$. Therefore, the general closed expression for ITH, is the result announced in Eq. 2. Expression for intra-tumor heterogeneity in Eq,2 for an exogenous cancer is expressible solely in terms of the probability of gene mutation $(p)$ and cell birth $(\alpha)$. It is worth reiterating that the simplicity of the analytic result makes it most useful in analyzing the experimental data.

\section{B. Comparison of theory to M-Seq data}

We calculated ITH of individual patients with skin [42], lung [43, 44, esophagus [45] or kidney [20] cancers from M-seq data (described in section II.A). In order to compare theory (Eq. 2) with M-Seq data, $x_{t}$ and $\alpha$, need to be evaluated for every patient specific M-seq data.

Estimating $\alpha$ : During tumor evolution, the cancer cells usually acquire between $(1-10)$ driver mutations 55$]$, which bestow fitness advantage to the deleterious cells. Assuming each driver mutation increases the birth probability by 0.01 and the number of driver mutations is 5 , we obtain $\alpha=0.55$.

Estimating $x_{t}$ : The parameter, $x_{t}$, can be directly calculated from the M-Seq data. Note, that $x_{t}$ refers to the probability that a gene loci is not mutated at $t$. In order to explain the calculation of $x_{t}$ for each patient, we use Figure 1b. All M-Seq readouts satisfy $0 \leq x_{t} \leq 1$. For the data in Figure 1 1 b, the total number of sites is $(n=83) \times 7=581$. From Figure 10 it follows that the total number of non-mutated sites in R2 is 37, R3 is 40, R4 is 42, R6 is 22 , R1 is $40, \mathrm{R} 5$ is 41 and R7 is 35 . The sum of these numbers $=257$, gives the total non-mutated sites in Figure $1 \mathrm{p}$, which unifies $x_{t}=\frac{257}{581}=0.44$. Similarly, we calculated the $x_{t}$ value for other patients. 
Using $\alpha=0.55$ and $x_{t}$, the ITH value can be evaluated (using Eq,2 for all the patients. Figure 4 , that compares the theoretical predictions and M-seq data, shows ITH for patients with exogenous cancers (i.e skin, lung and esophagus) is well captured by our theory. In contrast, the theory drastically overestimates the ITH value for kidney cancer, which is endogenous.

To quantify the accuracy of the theory in estimating the M-seq data, we use the Pearson correlation coefficient $(\rho)$. The value of $\rho$ for skin cancer is 0.97 with a $p$ value of $10^{-4}$, and 0.88 for lung cancer with a $p$ value of 0.02 (Figure 4 a). For the esophageal cancer, we did not calculate the $\rho$ value because the sample has only two patients. Surprisingly, the theory captures the heterogeneity for the two esophageal cancers accurately. We also compared the theoretical predictions with experiments for a different dataset, where the patients suffered from Non-Small Lung Cancer [44], in Figure $4 \mathrm{~b}$. The $\rho$ value for this dataset was 0.87 , which shows that the theory matches experiments.

The value of $\rho$ for kidney cancer is only 0.51 (Figure 4 ) with a $p$ value 0.13 , which implies that ITH behavior is not accurately predicted by the theory. Since kidney cancer is predominantly endogenous, we expect there might be some other factors that are not reflected in the theory, which uses only $\alpha$ and average mutation probability.

\section{Spatial distribution of tumor mutation burden (TMB)}

To discern the reason that the theory is effective in capturing ITH for exogenous cancers, we investigated the spatial distribution of TMB in distinct tumor regions. Figure 5 a and $5 \mathrm{~b}$ show the $x-y$ and $y-z$ cross-section of the simulated tumor. The colors indicate the TMB (number of genes with non-synonymous mutations) in each cell. The snapshots from tumor cross-sections show, in no uncertain terms, that TMB varies from cell to cell. To extract the spatial distribution of TMB across tumor regions, we divided the tumor into ten regions (similar to M-Seq data), each comprising of $\approx 5,000$ cells. The spatial variation of TMB is illustrated using the coefficient of variation $\left(c_{v}\right)$ of TMB across tumor regions,

$$
c_{v}=\frac{\sigma}{\mu}
$$

where $\sigma$ is the standard deviation of TMB, and $\mu$ is the mean TMB. A small (large) value of $c_{v}$ indicates low (high) variability. In Figure 55, we find that the $c_{v}$ value in simulations is 0.008 . We adopted a similar method to calculate the $c_{v}$ values from M-Seq data for the four cancer types.

Figure 5c shows the $c_{v}$ values for skin [42, lung [4], 44], esophageal 45] and kidney cancer [20]. The data shows that skin 42 has an average $c_{v} \approx 0.023$, implying that the extent of spatial variation in TMB is not significant. The data for Lung cancer[43] has an average $c_{v}$ of $\approx 0.086(\approx 8.6 \%)$ depicting that spatial distribution of TMB in lung cancer is less uniform than skin cancer. However, Figure 5 also shows that the $c_{v}$ value for lung cancer is large because of patient L002 $\left(c_{v} \approx 0.274\right)$, which might be an outlier among the six patients. The second Lung cancer dataset 44 has an average $c_{v}$ of $\approx 0.066(6.6 \%)$. Skin and lung cancers have approximately uniform spatial distribution of TMB, which resembles the simulation results. Esophageal cancer, which belongs to cancers predominantly caused by exogenous mutagens like skin and lung cancers, has the highest $c_{v}$ value $\left(c_{v} \approx 0.2\right)$. However, there are only two patient data, which prevents us from drawing general conclusions. Surprisingly, kidney cancer dataset 20 also has a very high $c_{v}$ value of $\approx 0.18$, which is approximately an order of magnitude higher than the $c_{v}$ for the skin-cancer dataset. The high TMB variation in endogenous cancers is not captured by the theory, which explains the low $\rho$ value for endogenous cancer. Finding ways to incorporate the heterogeneity in endogenous cancer, and connect it to TMB would be an interesting avenue for future research 56 .

\section{Spatial Variations in ITH}

In the previous sections, we dealt with the average values of intra-tumor heterogeneity. However, a lot of information is lost when considering only average measures of ITH. Most importantly, spatial variations in heterogeneity are smeared out. To illustrate the extent of spatial variations, we consider semi-infinite lattice, infinite lattice and $3 \mathrm{D}$ lattice geometry with the birth probability, $\alpha=1$. For the case of semi-infinite case, where growth occurs unidirectionally away from the origin, we begin with a cell at the origin at time $t=0$. Since $\alpha=1$, at every time step, only the cell at the boundary will divide. Therefore, the number of cells, $M(t)=t+1$. We evaluate the average heterogeneity as a function of both inter-cellular distance $(r)$ and time $(t)(G(r, p, t))$. 
For the case of semi-infinite $1 \mathrm{D}$ lattice, $G_{1 D, 1}(r, p, t)$ is given by

$$
G_{1 D, 1}(r, p, t)=\frac{1}{M(t)-r} \sum_{i=1}^{M(t)-r} 2 x_{t}\left(1-x_{t-i}\right)
$$

where $M(t)-r$ denotes the number of pairs of cells with inter-cellular distance $r$. In Eq. 11 the $2 x_{t}\left(1-x_{t-i}\right)$, is the effective average HD (see Eq. 21 in the Appendix) for a pair of cells at $t$ and $(t-i)$. Upon performing the summation, Eq. 11 becomes,

$$
G_{1 D, 1}(r, p, t)=2 x_{t}\left[1-\frac{x_{t}}{t-r}\left\{\frac{1}{p}\left(\frac{x_{r}}{x_{t}}-1\right)\right\}\right],
$$

where $x_{t}=(1-p)^{t}$ and $x_{r}=(1-p)^{r}$. Figure 6a shows that agreement between simulations and Eq. 12 is excellent. We observe that ITH is small for smaller inter-cellular distance because majority of cells that are just born are close to their parent, and hence, the daughter cells retain the character of the parent cells. We consider that since small distance incorporate recent birth events they have less heterogeneity compared to cells with larger inter-cellular distance.

For the infinite $1 \mathrm{D}$ lattice, where growth can occur on both the sides of the origin, $G_{1 D, 2}(r, p, t)$ is given as,

$$
G_{1 D, 2}(r, p, t)= \begin{cases}2 x_{t}\left[1-\frac{x_{t}}{2 t-r}\left\{\frac{2}{p}\left(\frac{x_{r}}{x_{t}}-1\right)+r\right\}\right], & \text { if } r<t \\ 2 x_{t}\left(1-x_{t}\right), & \text { if } r \geq t\end{cases}
$$

The average spatial and temporal variation of $G_{1 D, 2}(r, p, t)(\alpha=1)$ in Eq. 13 behaves differently depending on the condition $r<t$ and $r \geq t$. This is because in $1 \mathrm{D}$ infinite lattice there are 2 branches. Heterogeneity among cells with inter-cellular distance $r \geq t$, implies that the 2 cells belong to different branches. Therefore, they evolve independently. If $r<t$, we have to incorporate cells within a single branch as well as cells from the 2 different branches. Figure 6 $\mathrm{p}$ shows a good agreement between simulations and theory.

For the 3D lattice, it is difficult to get a closed form expression for the spatial dependence of heterogeneity because the number of branches $\propto 4 \pi R^{2}$, where $R$ is the radius of the tumor. Therefore, we just show simulation results. Figure 6 shows the dependence of heterogeneity as a function of inter-cellular distance. Even in this case, we observe that at large distances the heterogeneity saturates approximately to the value for the independent case.

Sub-Sample to Sub-sample variations in a single tumor: We resorted to 3D cellular automaton simulations to reveal the spatial variations in ITH using $\alpha=0.55$ and $p=0.003$. Figure 7 illustrates the massive sample to sample Hamming distance variations in the tumor. Figure $7 \mathrm{a}$ shows the Hamming distance map between 2,000 cells, represented as a $2,000 \times 2,000$ matrix. The matrix shows that different cell pairs have different HD values, representing different levels of heterogeneity. The zoomed in matrix of size $100 \times 100$ in Figure $7 \mathrm{~b}$, shows the heterogeneity among cell pairs at a finer resolution. It is clear that the HD values change dramatically varying from 0 to 0.5 . The HD distance in Figure 7 a gradually changes as cells from different regions are sampled, as can seen from Figure 7 k (zoomed in Figure 7d) and 7e (zoomed in Figure 7f). Hamming distance matrices in Figures 7k and 7e, are calculated for cells which are approximately 20 and 27 lattice units away from the center of the tumor. The color pattern, quantifying the magnitude of Hamming Distance, gradually changes from yellowish to green (Figures $7 \mathrm{k}, 7 \mathrm{k}$ and $7 \mathrm{p}$ ). Taken together, Figure 7 shows the rich heterogeneous spatial patterns that emerges depending on where we probe the tumor. The ITH, provides insights into the complexity of the tumor evolution. The results in Figure 7 suggest that if the number of biopsied regions increases, it would reveal far greater changes in $H D$ than is portrayed in Figure 1 . Of course, there are serious practical limitations when data from human patients is sought.

Figure 8 shows the colorful pattern obtained when the cells, located $\sim 20$ lattice units away from the tumor centre, are painted according to their $H D$ values with respect to the cell at the tumor center. Though tantalizingly beautiful, it shows that quantifying intra-tumor heterogeneity, represented as a HD map, is a challenging problem.

\section{DISCUSSION \& CONCLUSION}

We developed a statistical mechanical theory, supplemented by simulations, for spatio-temporal variations in ITH associated with cancers. The resulting theoretical expressions are used to analyze M-Seq data on four cancer types obtained from biopsies from multiple regions in a single solid tumor. The agreement between theory and experiments is good for exogenous cancers (skin and lung) but not so for kidney cancers, which is endogenous. The most likely 
explanation is that factors besides cell division probability $(\alpha)$ and mutation probability $(p)$, which are the only ingredients in the theory, are relevant. At present, it is unclear how unknown factors could be taken into account.

Our theory, based on the premise that neutral evolution is valid [57 61], uses Hamming Distance $(H D)$ as a measure to quantitatively describe genetic variations in M-Seq data. We show that ITH can be factored into a product of two terms, $G(p, t)$ and $F(\alpha)$, where $G(p, t)$ is the ITH for $\alpha=1$, and $F(\alpha)$ is the scale factor that takes into account that $\alpha$ $(0.5<\alpha<1)$ is less than unity. We discovered that $G(p, t)$ depends on the geometry in which the tissue is embedded. Surprisingly, we find in three-dimensions, $G(p, t)=2 x_{t}\left(1-x_{t}\right)$ which coincides with the result for cells that evolve independently. The scale factor $F(\alpha)$ depends linearly on $\alpha, F(\alpha)=(0.2 \alpha+0.77)$. It is remarkable that the final expression of ITH (Eq 2), whose validity is confirmed by simulations, accurately explains the results from M-Seq data obtained from patient data. The theory accurately quantifies ITH in exogenous cancers. The theory predicts that endogenous cancer has one order of magnitude higher $c_{v}$ value compared to exogenous case which explains the lack of success in explaining ITH in edogenous cancers.

The cellular and automaton type simulations, which were carried out to confirm the theoretical predictions, vividly illustrate the pervasive nature of ITH. On all length scales in the in silico tumor, we find that there are substantial ITH variations, as vividly illustrated in Figure 7. The patterns in the HD map suggests that there are dramatic changes over the same size of tumor regions, as illustrated in Figures $7 \mathrm{~b}, 7 \mathrm{~d}$, and $7 \mathrm{f}$. We find it remarkable that the $2 \mathrm{D}$ matrices associated with the HD map reveal striking dissimilarity, as is evident from Figure 8 and additional plots in the Figure S13. These figures show visually that the ITH in distinct regions are vastly different, suggesting the mere coarse-grained representation available from experiments (Figure 1 b). We should caution the reader that the large scale heterogeneity that is visualized in the simulations may not be revealed (or even present) in solid tumors because it would ultimately require sequencing at the single cell resolution 62. In addition to spatial heterogeneity distribution, the heterogeneity distribution also evolves in time as is reflected in Figure S14.

In addition to the genetic heterogeneity discussed above, phenotypic variations 63, 64, of cancer cells are also important, which is crucial in devising modern personalized cancer therapeutics [65]. Recent advancements in imaging modalities have helped unearth heterogeneous physical characteristics at the single cell resolution in three-dimensional cell collectives [66 68]. Our previous studies have shown that cell-division and apoptosis induced self-generated forces give rise to phenotypic diversity within a tumor [69 75]. In principle, the current theoretical framework, could be extended to estimate the ITH for both genetic and phenotypic heterogeneity of evolving tumors.

Acknowledgements This work is supported by a grant from the National Science Foundation (PHY 17-08128 and PHY-1522550). Additional support was provided by the Collie-Welch Reagents Chair (F-0019).

\section{APPENDIX}

Motivated by the data in Figures S2-S6 in SI, we developed a statistical mechanical (or probabilistic) theory for quantifying ITH, which is described in the main text. Here, we provide the technical details.

Heterogeneity in independently evolving cells: Consider two cells, 1 and 2, which evolve independently (Figure S7a in SI). The elements in the string $X^{1}$ and $X^{2}$, which are the genes, are initialized to zero at $t=0$. This implies that there are no mutations at $t=0$. Given the rules for acquiring mutations (described in the Methods section in the main text), we evaluate the Hamming distance $\left(\left\langle H D\left[X^{1}(t), X^{2}(t)\right]\right\rangle\right)$, where $\langle\ldots\rangle$ represents the ensemble average. We generate an ensemble of DNA strings with mutations from an evolutionary trajectory over a certain time. At each time step, a DNA string associated with a cell, can acquire mutations. The set of mutations acquired at the end of the evolutionary period constitutes the ensemble.

The Hamming distance $\left(\left\langle H D\left[X^{1}(t), X^{2}(t)\right]\right\rangle\right)$ is,

$$
\left\langle H D\left[X^{1}(t), X^{2}(t)\right]\right\rangle=\sum_{k=1}^{n} \frac{k}{n}\left\{\mathbb{P}\left[H D\left[X^{1}(t), X^{2}(t)\right]=\frac{k}{n}\right]\right\}
$$

$\mathbb{P}\left[H D\left[X^{1}(t), X^{2}(t)\right]=\frac{k}{n}\right]$ is the probability that the HD between cell 1 and 2 is $\frac{k}{n}$ at $t$. We divide by the string length, $n$, because the sites on the string are independent of each other. Moreover, in the M-seq experiments the string length $n$, varies from patient to patient. Therefore, dividing by $n$ enables us to treat each patient data on the same footing. 
The expression for the right hand side of Eq. 14 is,

$$
\begin{aligned}
\mathbb{P}\left[H D\left[X^{1}(t), X^{2}(t)\right]=\frac{k}{n}\right] & \\
& =\sum_{\substack{z_{0}+z_{1}+z_{2}+z_{12}=n \\
z_{1}+z_{2}=k}} \frac{n !}{z_{0} ! z_{1} ! z_{2} ! z_{12} !}(1-p)^{t\left(2 z_{0}+z_{1}+z_{2}\right)}\left[1-(1-p)^{t}\right]^{2 z_{12}+z_{1}+z_{2}},
\end{aligned}
$$

where $z_{0}$ is the number of common sites in $X^{1}$ and $X^{2}$ that are not mutated, and $z_{12}$ denotes all the common sites in $X^{1}$ and $X^{2}$ that are mutated. The number of sites where $X^{1}$ is mutated but the corresponding site in $X^{2}$ is not mutated is $z_{1}$. Similarly, $z_{2}$ denotes the number of sites where $X^{2}$ is mutated but the corresponding site in $X^{1}$ is not mutated. We also have the constraints that $z_{1}+z_{2}=k$, and $z_{0}+z_{1}+z_{2}+z_{12}=n$. In Eq. 15 the term $(1-p)^{t\left(2 z_{0}\right)}$ accounts for the probability that $z_{0}$ common sites are not mutated, which is the product $(1-p)^{t\left(z_{0}\right)}(1-p)^{t\left(z_{0}\right)}$. Similarly, in Eq. 15 . $\left(1-(1-p)^{t}\right)^{2 z_{12}}$ is the probability that $z_{12}$ common sites are mutated $\left[1-(1-p)^{t}\right]^{z_{12}}\left[1-(1-p)^{t}\right]^{z_{12}}=\left[1-(1-p)^{t}\right]^{2 z_{12}}$.

We can calculate the right hand side of Eq. 15 to get,

$$
\mathbb{P}\left[H D\left[X^{1}(t), X^{2}(t)\right]=\frac{k}{n}\right]=\left(\begin{array}{l}
n \\
k
\end{array}\right)\left\{2 x_{t}\left(1-x_{t}\right)\right\}^{k}\left\{x_{t}^{2}+\left(1-x_{t}\right)^{2}\right\}^{n-k},
$$

where $x_{t}=(1-p)^{t}$ is the probability that a site has not been mutated till $t$. We substitute Eq. 16 in the R.H.S of Eq. 14 and obtain,

$$
\left\langle H D\left[X^{1}(t), X^{2}(t)\right]\right\rangle=\sum_{k=1}^{n} \frac{k}{n}\left(\begin{array}{l}
n \\
k
\end{array}\right)\left\{2 x_{t}\left(1-x_{t}\right)\right\}^{k}\left\{x_{t}^{2}+\left(1-x_{t}\right)^{2}\right\}^{n-k} .
$$

Surprisingly, equation 17 reduces to a very simple form given by,

$$
\left\langle H D\left[X^{1}(t), X^{2}(t)\right]\right\rangle=2 x_{t}\left(1-x_{t}\right) .
$$

Eq. 18 is the average heterogeneity among independently evolving cells. In our case, this type of evolution refers to cells on two different 1D semi-infinite branches. Figure S7b in the SI shows that the theoretical prediction given in $\mathrm{Eq} 18$ is in excellent agreement with the simulation results.

Heterogeneity for non-independent evolving cells: We now consider a scenario when two cells have a common ancestor at time, $t>0$. Let us consider the case when cell 1 evolves in time, acquiring exogenous mutations, from $t=0$ till $t$. After time $t$, it divides and gives birth to cell 2. During the birth process, the information contained in string $X^{1}$ is copied to string $X^{2}$. Figure S8 in the SI illustrates the correlated evolutionary dynamics.

Our goal is to evaluate the average heterogeneity $\left(\left\langle H D\left[X^{1}(t+s), X^{2}(t+s)\right]\right\rangle\right)$ between cells 1 and 2 at a later time $=t+s$. As before, $\langle\ldots\rangle$ represents the ensemble average. Following the same method used to derive Eq. 18. we obtain,

$$
\mathbb{P}\left[H D\left[X^{1}(t+s), X^{2}(t+s)\right]=\frac{m}{n}\right]=\left(\begin{array}{c}
l \\
m
\end{array}\right)\left\{2 x_{s}\left(1-x_{s}\right)\right\}^{m}\left\{x_{s}^{2}+\left(1-x_{s}\right)^{2}\right\}^{l-m},
$$

where $x_{s}=(1-p)^{s}$, and $l$ is the number of non-mutated sites in string $X^{1}(t)$. It can be shown that $\langle l\rangle=n(1-p)^{t}$. Using a similar expression, as in Eq. 14, we can write,

$$
\left\langle H D\left[X^{1}(t+s), X^{2}(t+s)\right]\right\rangle=\sum_{m=1}^{l} \frac{m}{n}\left\{\mathbb{P}\left[H D\left[X^{1}(t), X^{2}(t)\right]=\frac{m}{n}\right]\right\} .
$$

By substituting Eq. 19 in the R.H.S of Eq. 20, we obtain,

$$
\left\langle H D\left[X^{1}(t+s), X^{2}(t+s)\right]\right\rangle=2 x_{s}\left(1-x_{s}\right) x_{t} .
$$

The above equation can also be written as $\left\langle H D\left[X^{1}(t+s), X^{2}(t+s)\right]\right\rangle=2 x_{t+s}\left(1-x_{s}\right)$. Equation 21, represents the average heterogeneity between two cells which had common evolution till time $t$ but evolved independently for the subsequent time interval, $s$. It can be shown from the expression given above that if we set $t=0$, we get back the equation for independent evolution, derived in Eq.18. The result in Eq. 21 is very interesting because it shows that the temporal correlations between the two cells arises because of the copying mechanism during cell-division, which also implies that the heterogeneity is smaller compared to the independently evolving cells (Eq.18). 
Branching increases intra-tumor heterogeneity: The heterogeneity measure between cells that evolve independently and in a correlated manner, allows us to compute the average heterogeneity withing an evolving tumor for $\alpha=1$, which we denote as $G(p, t)$. We present the results for $G(p, t)$ for various physical geometries: $G_{1 D, 1}(p, t)$ for semi-infinite lattice , $G_{1 D, 2}(p, t)$ for infinite lattice, $G_{2 D}(p, t)$ for $2 \mathrm{D}$ lattice, and $G_{3 D}(p, t)$ for $3 \mathrm{D}$ lattice with birth probability $\alpha=1$. We consider these geometries because the number of branches in an evolutionary tree depends on its geometrical structure. The M-Seq data shows that the phylogeny tree of a tumor consists of many branches [19], in contrast to the linear evolution model suggested by Nowell 15. Thus, by considering different physical geometries representing the branches, we can probe the role of branching on ITH.

To calculate $G(p, t)$, we first introduce $F\left(r_{1}, r_{2}, t\right)$, which is the average heterogeneity for pair of cells at distance $r_{1}$ and $r_{2}$ from the origin at time $t$. Without loss of generality, we assume that $r_{1} \geq r_{2}$. The general expression for $F\left(r_{1}, r_{2}, t\right)$ for any geometry is given by,

$$
F\left(r_{1}, r_{2}, t\right)=P_{b r}\left\{2 x_{t}\left(1-x_{t-r_{2}}\right)\right\}+\left(1-P_{b r}\right)\left\{2 x_{t}\left(1-x_{t}\right)\right\}
$$

where $P_{b r}$ is the probability that both the cells belong to the same branch of the evolutionary tree. For the 1D semi-infinite lattice, $P_{b r}=1$ because there is only one branch. For the 1D infinite lattice, $P_{b r}=\frac{1}{2}$. For a $2 \mathrm{D}$ lattice, $P_{b r}=\frac{\left(\pi r_{1}^{2}\right) r_{1}}{\left(\pi r_{1}^{2}\right)\left(\pi r_{1}^{2}\right)}=\frac{1}{\pi r_{1}}$, and for the 3D lattice, $P_{b r}=\frac{\left(\frac{4}{3} \pi r_{1}^{3}\right) r_{1}}{\left(\frac{4}{3} \pi r_{1}^{3}\right)\left(\frac{4}{3} \pi r_{1}^{3}\right)}=\frac{1}{\frac{4}{3} \pi r_{1}^{2}}$. In 2D and 3D, the number of cells within a radius $r_{1}$ is proportional to $\pi r_{1}^{2}$ and $\frac{4}{3} \pi r_{1}^{3}$ respectively, and the number of cells that lie on the same branch is proportional to $r_{1}$ as long as the branches are linear. Therefore, we obtain the above expressions for $P_{b r}$ in $2 \mathrm{D}$ and 3D. Note that to arrive at the expression for $P_{b r}$, we have assumed that the evolutionary tree is comprised of many semi-infinite lattices with zero curvature. It is important to note that for $2 \mathrm{D}$ and $3 \mathrm{D}$ case, $P_{b r} \rightarrow 0$ for $r_{1}>>1$. Interestingly, $F\left(r_{1}, r_{2}, t\right)$ does not depend on $r_{1}$ but only on $r_{2}$ for $1 \mathrm{D}$ semi-infinite or infinite lattice. Eq. 22 has two parts, which we derived in Eqs. 18 and 21. The first part corresponds to cells that undergo correlated evolution while the second part refers to cells undergoing independent evolution. Therefore, in all the possible geometries, $F\left(r_{1}, r_{2}, t\right)$ takes the following form,

$$
F\left(r_{1}, r_{2}, t\right)= \begin{cases}2 x_{t}\left(1-x_{t-r_{2}}\right), & \text { 1D semi-infinite } \\ \frac{1}{2} 2 x_{t}\left(1-x_{t-r_{2}}\right)+\left(1-\frac{1}{2}\right) 2 x_{t}\left(1-x_{t}\right), & \text { 1D infinite } \\ \frac{1}{\pi r_{1}} 2 x_{t}\left(1-x_{t-r_{2}}\right)+\left(1-\frac{1}{\pi r_{1}}\right) 2 x_{t}\left(1-x_{t}\right), & 2 \mathrm{D} \\ \frac{1}{\frac{4}{3} \pi r_{1}^{2}} 2 x_{t}\left(1-x_{t-r_{2}}\right)+\left(1-\frac{1}{\frac{4}{3} \pi r_{1}^{2}}\right) 2 x_{t}\left(1-x_{t}\right), & 3 \mathrm{D}\end{cases}
$$

To compute $G(p, t)$ (Eq.(2) in the main text), we need to integrate $F\left(r_{1}, r_{2}, t\right)$ over space with appropriate normalization. The expression for $G(p, t)$ in different geometries are given by,

$$
G(p, t)= \begin{cases}G_{1 D, 1(2)}(p, t)=\frac{\int_{1}^{R} \int_{1}^{r_{1}} F\left(r_{1}, r_{2}, t\right) d r_{2} d r_{1}}{\int_{1}^{R} \int_{1}^{r_{1}} d r_{2} d r_{1}}, & \text { if 1D semi-infinite or infinite lattice. } \\ G_{2 D}(p, t)=\frac{\int_{1}^{R} \int_{1}^{r_{1}} r_{1} r_{2} F\left(r_{1}, r_{2}, t\right) d r_{2} d r_{1}}{\int_{1}^{R} \int_{1}^{r_{1}} r_{1} r_{2} d r_{2} d r_{1}}, & \text { if } 2 \mathrm{D} \text { lattice. } \\ G_{3 D}(p, t)=\frac{\int_{1}^{R} \int_{1}^{r_{1}} r_{1}^{2} r_{2}^{2} F\left(r_{1}, r_{2}, t\right) d r_{2} d r_{1}}{\int_{1}^{R} \int_{1}^{r} r_{1}^{2} r_{2}^{2} d r_{2} d r_{1}}, & \text { if 3D lattice. }\end{cases}
$$

In the above equation, $R$ is the tumor radius in all the geometries except semi-infinite lattice (for semi-infinite case $R$ is the length of the tumor). On integrating the above equations in Eq. 24 using Mathematica, $G(p, t)$ in all the geometries is given by,

$$
\begin{aligned}
& G_{1 D, 1}(p, t)=\frac{2(1-p)^{t}}{(R-1)^{2}}\left((R-1)^{2}-\frac{(1-p)^{t-R-1}}{\log ^{2}(1-p)}\left\{2(1-p)^{R}[(R-1) \log (1-p)-1]-2(p-1)\right\}\right) \\
& G_{1 D, 2}(p, t)= \\
& \frac{2(1-p)^{t}}{(R-1)^{2}}\left(\frac{(1-p)^{t-R-1}}{\log ^{2}(1-p)}\left\{(1-p)^{R}+(p-1)-(R-1)(1-p)^{R} \log (1-p)\right\}-\frac{1}{2}(R-1)^{2}\left[(1-p)^{t}-2\right]\right)
\end{aligned}
$$




$$
\begin{aligned}
& G_{2 D}(p, t)= \\
& \begin{array}{r}
\frac{8(1-p)^{t}}{\pi\left(R^{2}-1\right)^{2}}\left\{-\frac{1}{12}(R-1)^{2}\left[3 \pi(R+1)^{2}\left((1-p)^{t}-1\right)-4(R+2)(1-p)^{t}\right]\right. \\
-\frac{(1-p)^{t-R-1}}{\log ^{3}(1-p)}\left(-2(p-1)[2+R \log (1-p)]+2(1-p)^{R}[-2+\right. \\
\log (1-p)\{(R-1) \log (1-p)+(R-2)\}])\}
\end{array}
\end{aligned}
$$

$$
\begin{aligned}
& G_{3 D}(p, t)= \\
& \frac{3(1-p)^{t}}{\pi\left(R^{3}-1\right)^{2}}\left(-\frac{2}{3} \pi\left(R^{3}-1\right)^{2}\left[(1-p)^{t}-1\right]+\frac{3}{4}(R-1)^{2}(1-p)^{t}\left(R^{2}+2 R+3\right)\right. \\
& -\frac{(1-p)^{t-R-1}}{\log ^{4}(1-p)}\left[9(1-p)^{R}\{-6+\log (1-p)(2(R-3)+\log (1-p)\{2 R-3+(R-1) \log (1-p)\})\}\right. \\
& -9(p-1)[6+R \log (1-p)\{R \log (1-p)+4\}]])
\end{aligned}
$$

Comparison beween Theory and Simulations: To validate the theoretical predictions, we compared the results in Eqs. 25, 26 and 28 using simulations. Figure 2d shows excellent agreement between equation (25) and simulations for the 1D semi-infinite lattice. The 1D semi-infinite lattice can be thought of as a branch in the $3 \mathrm{D}$ evolutionary tree. Therefore, as expected the heterogeneity among cells within the same branch is smaller compared to cells that evolve independently. The maximum average heterogeneity when the cells evolve independently, as can be gleaned from Eq. [18), is 0.5 whereas the maximum average heterogeneity among cells within a branch is $\approx 0.36$. The substantial reduction in heterogeneity occurs because of correlations among the cells arising from copying genetic information from parent to child during cell division.

Similarly, for tumor evolution in 1D infinite lattice, the agreement between theory (Eq. 25) and simulations is excellent (Figure 2). The 1D infinite lattice comprises of 2 branches with an angle of $180^{\circ}$ between the two. The nodes on the two branches are completely independent of one another. However, due to the non-independent evolution of cells within the two branches, the heterogeneity is small compared to the independent evolution case. The maximum heterogeneity is $\approx 0.43$ (Eq. 25 ).

Evolution of tumor in 3D lattice is the most interesting case, and is most relevant. Figure $2 \mathrm{f}$ again shows good agreement between theory (equation 28) and simulations. It is surprising that in 3D, the heterogeneity is similar to the case of independent evolution. This can be understood with the help of equation 22 with $P_{\text {branch }} \rightarrow 0$. In this limit, the average heterogeneity behaves like the case for independent evolution (i.e $2 x_{t}\left(1-x_{t}\right)$ ). The maximum heterogeneity in this case is $\approx 0.5$.

Having shown that our theoretical results are consistent with simulations, we compare the theoretical predictions for semi-infinite, infinite and 3D lattice together. We can clearly see from Figure S9d that $G_{1 D, 1}(p, t)<G_{1 D, 2}(p, t)<$ $G_{3 D}(p, t)$. The inequality follows because as we increase the dimensionality, we give way to more branches which undermines the effect of reduction of heterogeneity due to copying of genetic information during cell division. Therefore, $G_{3 D}(p, t)$ is approximately similar to the case of independent evolution. 


\section{a)}

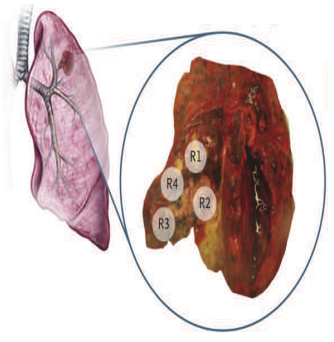

b)

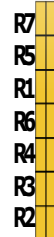

\section{c)}

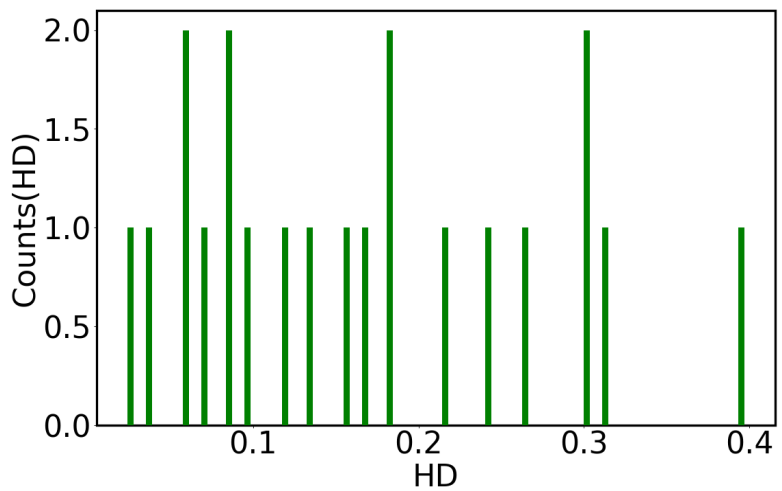

d)

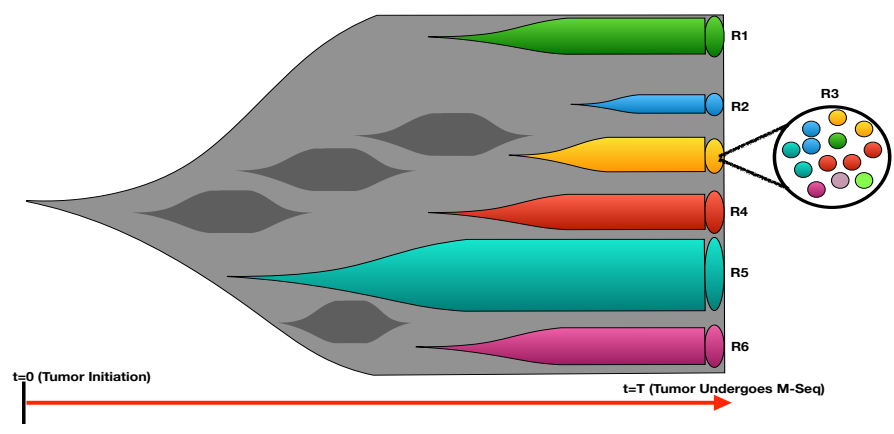

Figure 1: Schematic of Multiregion Sequencing (M-Seq) (a) A tumor sample (dark red) located in the lung (right lobe) of a cancer patient [4]. An enlarged view, depicted in the oval, is on the right. The labels R1, R2, R3 and R4 are the regions where the cells were extracted by biopsy and whole exome sequencing, referred to as Multiregion Sequencing (M-Seq), is performed. (b) A typical readout of the M-Seq experiment [20] for a kidney cancer patient where M-Seq in seven regions were conducted. In this heat map representation, the presence of mutated (non-mutated) gene in a region is denoted as a yellow (blue) box. The number of columns represent the total number of uniquely mutated genes $(n)$ in the entire tumor ( $n=83$ in this case). In the row adjacent to the heat map, the mutated genes are listed and the probable driver mutations are displayed in magenta. The rows represents the seven regions that were sampled.(c) Distribution of the Hamming Distance (HD) for the M-Seq data in (b). The y-axis (x-axis) shows the counts (HD values). Sum of the counts $=C_{2}^{7}=21$, the total number of region pairs for M-Seq data in (b). (d) A schematic for neutral evolution for cancer. The regions R1 to R6 with different colors represent the distinct genetic composition measured using M-Seq. The schematic represents neutral evolution because the size of different regions is proportional to their lifetime, cyan (blue) region being the biggest. We zoomed in on R3 to show the distinct genetic makeup of the cells. The dark grey lineages are do not survive during the course of tumor evolution. 
bioRxiv preprint doi: https:/doi org/10.1101/2022.02 19.481164; this version posted February $20,2022$. The copyright holder for this preprint (which was not certified by peer review) is the author/funder, who has granted bioRxiv a license to display the preprint in perpetuity. It is made available under aCC-BY-ND 4.0 International license.

a)

(1)

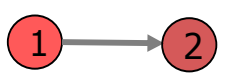

$t=0$ $t=1$

b)

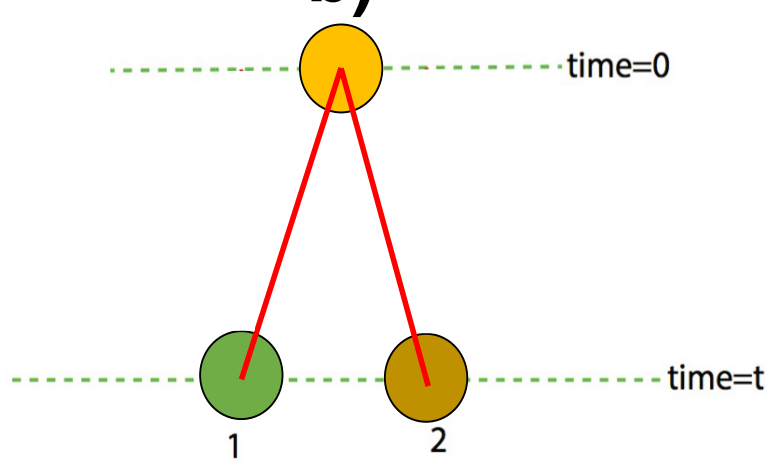

Independent Evolution

d)

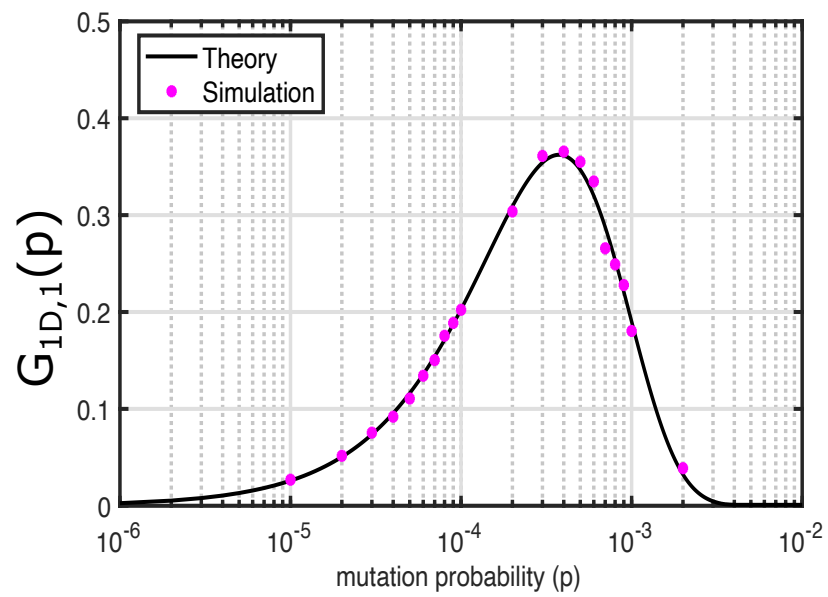

(3)
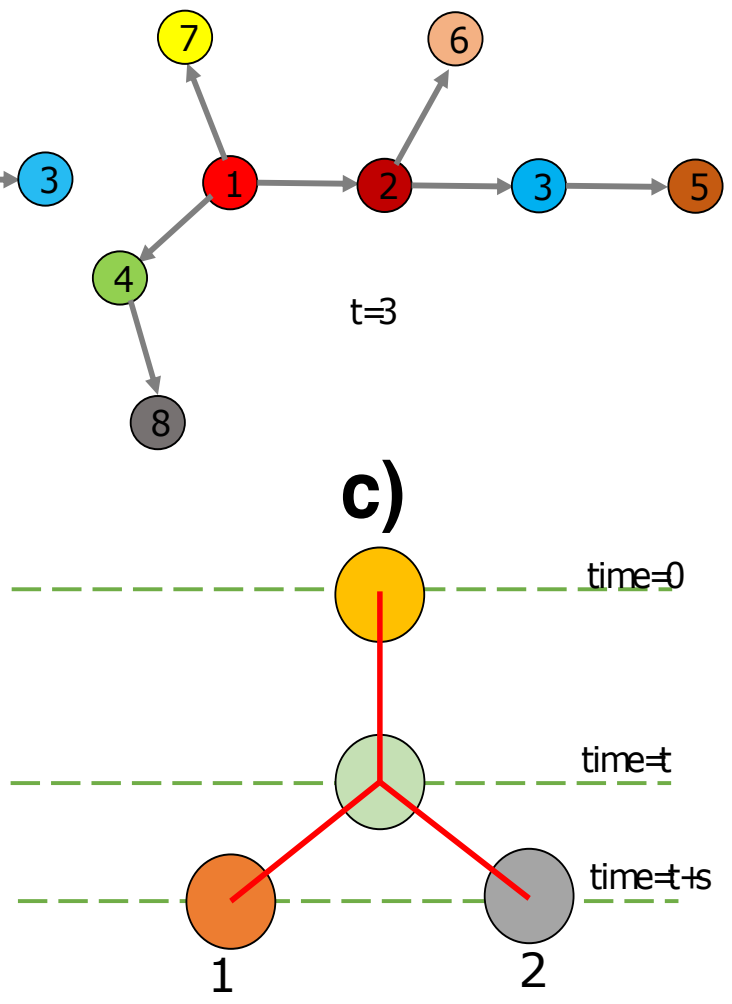

e)

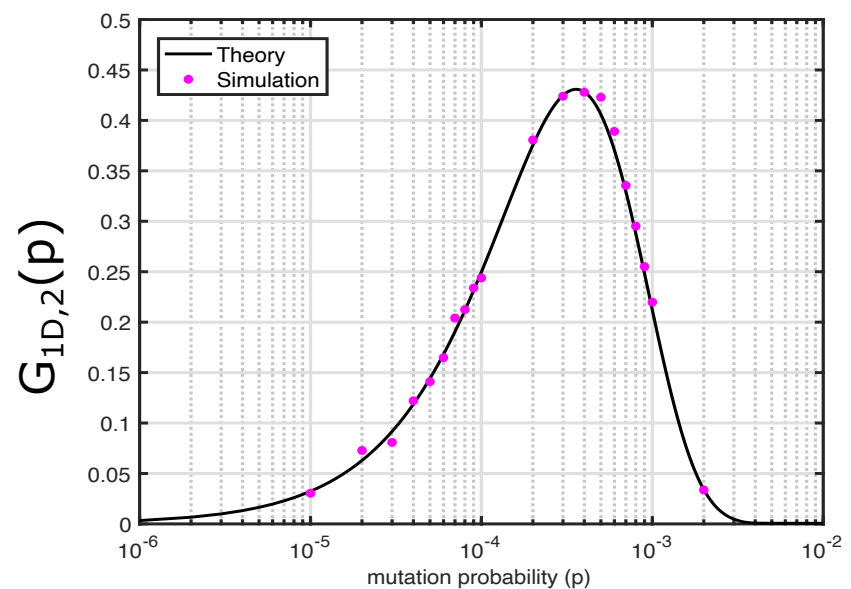

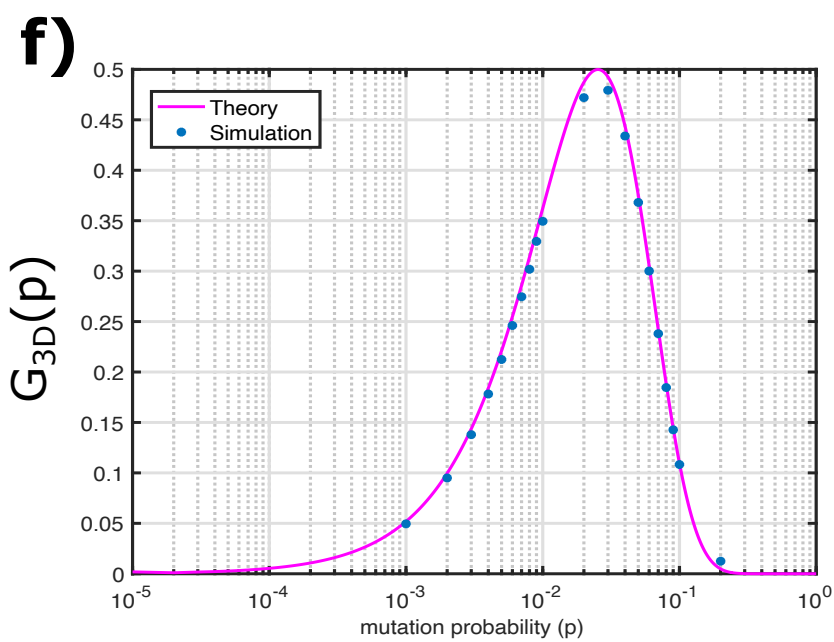

Figure 2: (Contined on the following page) 
Figure 2: Calculation of $\mathbf{G}(\mathbf{p}, \mathbf{t})$ for, $\alpha$, the birth probability set to unity. (a) A schematic of evolutionary tree at time, $t=0,1,2$ and 3. The cell colors (only for illustration purpose) gradually changes from transparent to opaque as they acquire mutations. The directed edges denote the child-parent relationship between two cells. For instance, the edge from $3 \rightarrow 5$ indicates that cell 5 was born from cell 3 . The evolutionary tree comprises of several branches. A branch is a unique path traversed from the origin (cell 1) to any leaf node (no directed edge from a node) by following the directed edges. In Figure 2 a, $1 \rightarrow 2 \rightarrow 3 \rightarrow 5$ is the longest branch at $\mathrm{t}=3$. (b) Cartoon depicting independent evolution of two cells (green and brown) labeled as 1 and 2. The two cells are normal at time $t=0$ and evolve independently acquiring mutations. (c) Schematic of correlated evolution of 2 cells (grey and orange). Evolution begins at $t=0$ with a single normal cell (yellow). It evolves till $t$, acquiring mutations (light green) in the process. At $t$, the cell gives birth to a daughter cell. After $t$, the 2 cells evolve independently for the next $s$ time steps. By correlated evolution we mean that the two cells have a common ancestor. In this instance, the common ancestor for the orange and grey cells at $t+s$ is the green cell at $t$. (d) Average ITH within an evolving tumor in 1D semi-infinite lattice $\left(G_{1 D, t}(p, t)\right)$. The dots in magenta correspond to simulations with $\alpha=1$ calculated by evolving for $t=2,000$ time steps. The black line corresponds to Eq. 25 in the SI with $R=t=2,000$. (e) Same as (d) but in 1D infinite lattice $\left(G_{1 D, 2}(p, t)\right)$. The black line is a plot of Eq. 26 in the SI with $R=t=2000$. (f) Same as $(\mathrm{d})$ but in 3D lattice $\left(G_{3 D}(p, t)\right)$ for $t=27$. The systems evolution was carried out for 27 time steps. The magenta line corresponds to Eq. 7 with $R=t=27$. 

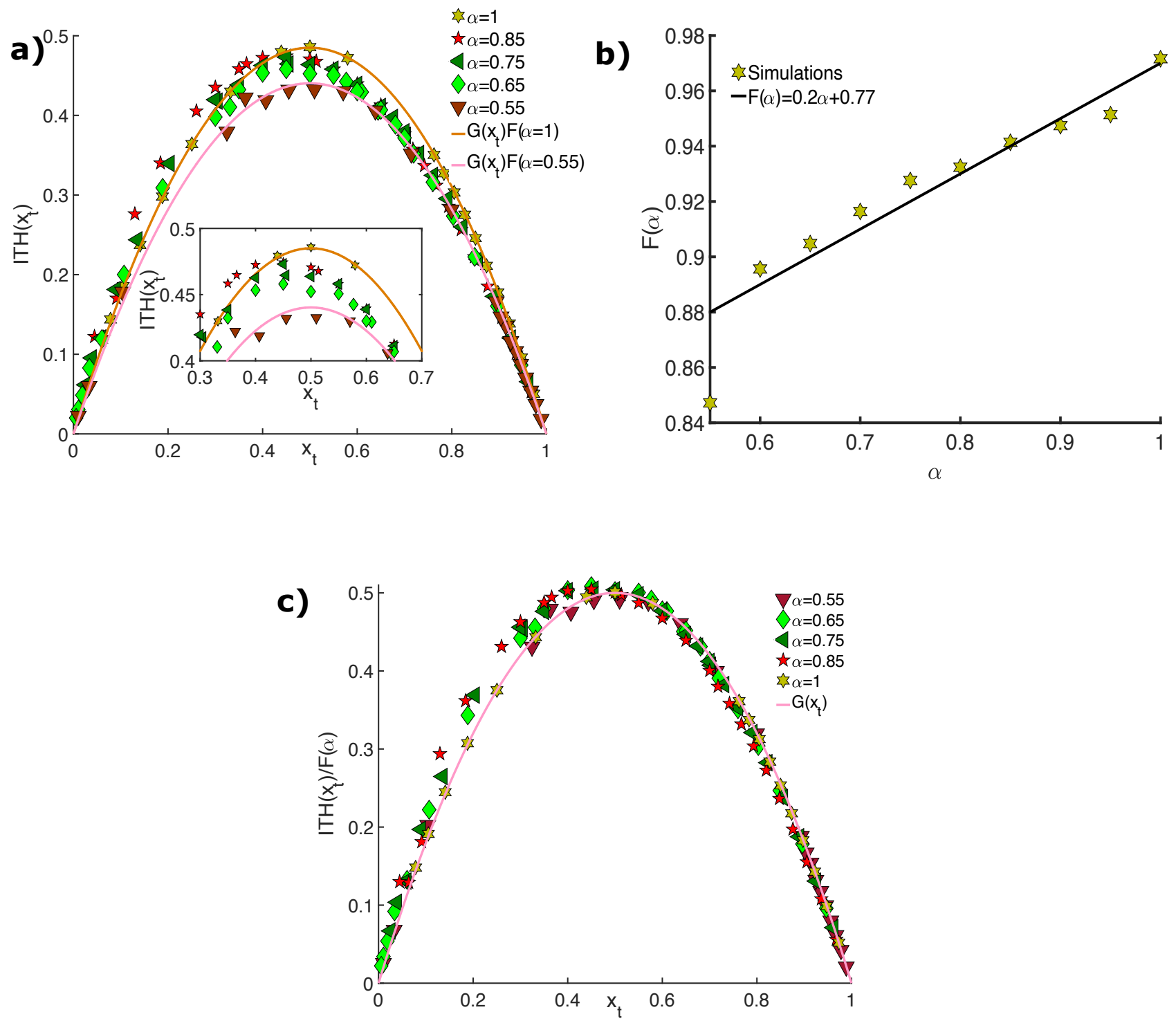

Figure 3: Theoretical Predictions for Intra-tumor Heterogeneity (a) ITH (Eq. 2) as a function of $x_{t}$ (probability a gene is not mutated) at $t$. Simulation results are shown for five values of $\alpha=1,0.85,0.75,0.65,0.55$, the birth probability. Reduction in $\alpha$ decreases the peak value of ITH, as is clear from the inset. (b) Plot of $F(\alpha)$ vs $\alpha . F(\alpha)=\frac{I T H\left(x_{t}=0.5\right)}{G_{3 D}\left(x_{t}=0.5\right)}$, was fit to a line $\left.(F(\alpha)=0.2 \alpha+0.77)\right)$, shown in black. (c) $\frac{I T H}{F(\alpha)}$, as a function of $x_{t}$ shows the collapse of the data sets onto a master curve, $G\left(x_{t}\right)=2 x_{t}\left(1-x_{t}\right)$. 

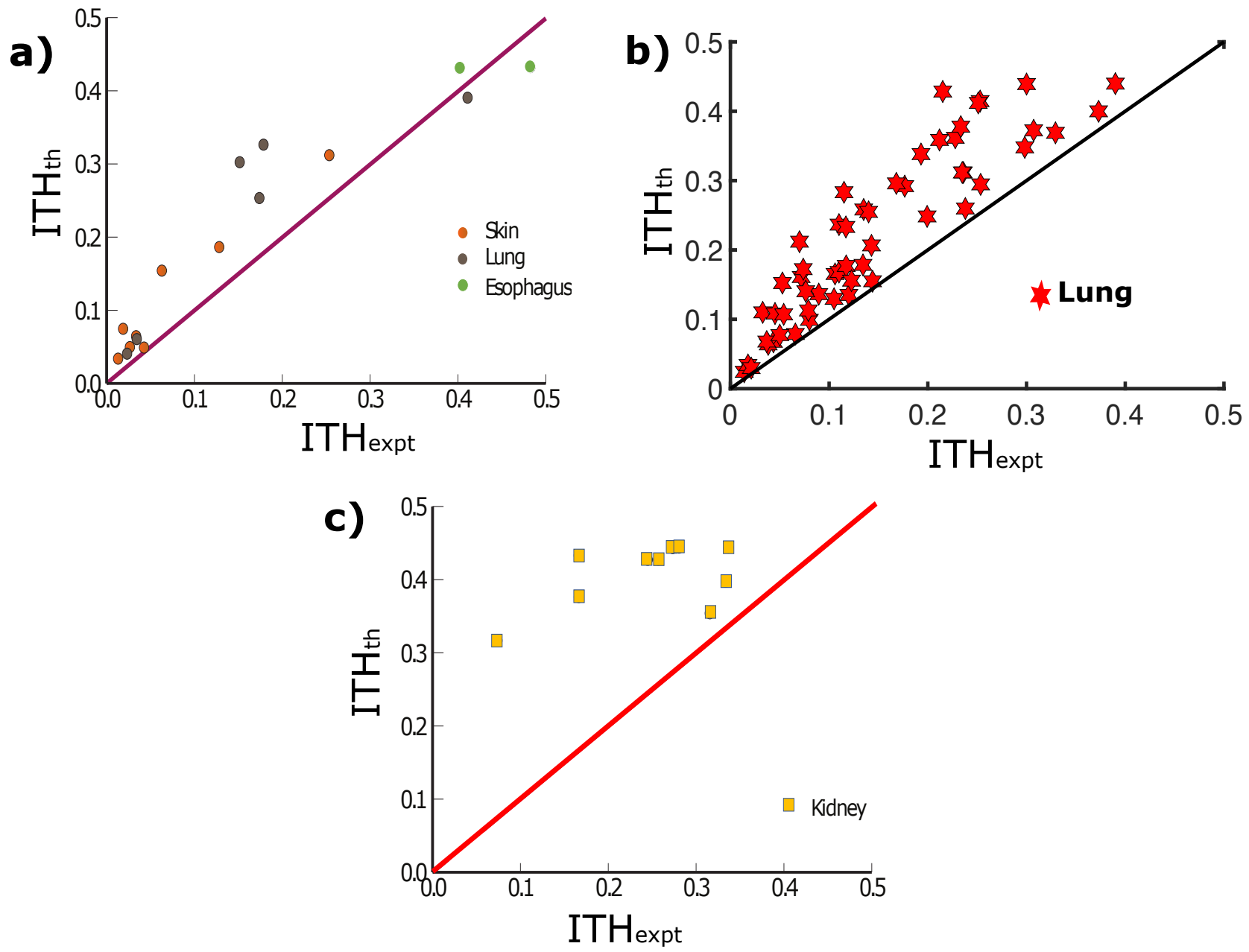

Figure 4: Comparison between theory and experiments (M-seq data) (a) The orange, brown and green colors represent skin, lung, esophageal cancer respectively. The maroon straight line represents perfect linear relation between theory and experiments. The theory captures the heterogeneity of exogenous cancers (skin, lung, esophagus) reasonably accurately. The value of $\rho$ (the Pearson Correlation coefficient) for skin cancer is 0.97 with a $p$ value of $10^{-4}$ and it is 0.88 for lung cancer with a $p$ value of 0.02 . (b) Same as (a) except the plot is for lung cancer dataset from Jamal et.al 44 . The value of $\rho$ for this dataset is 0.87 . (c) ITH for the endogenous kidney cancer, shown in yellow squares, which is an endogenous cancer is not captured well by the theory. For kidney cancer, the value of $\rho$ is 0.5 with a $p$ value 0.13 . 


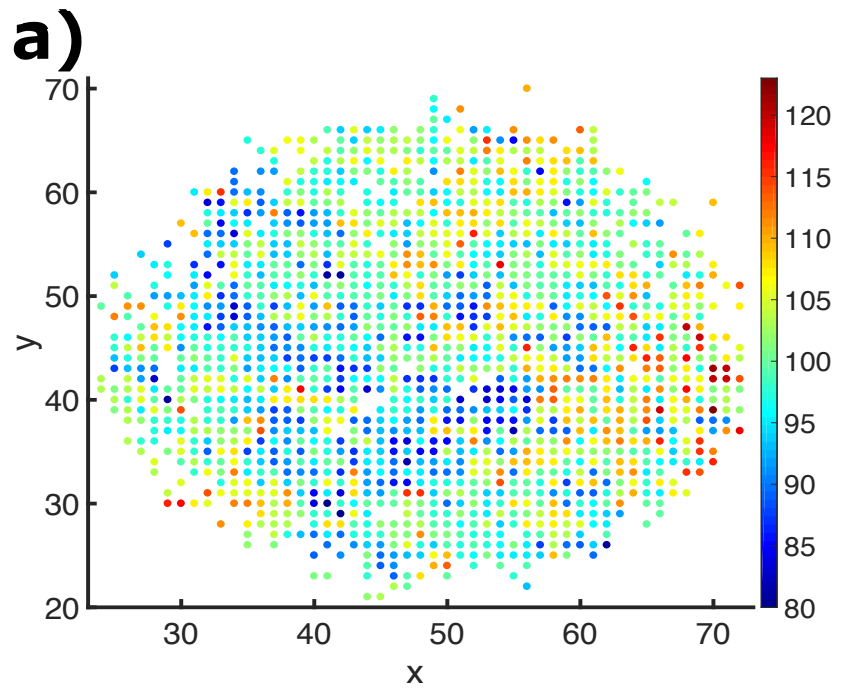

b)
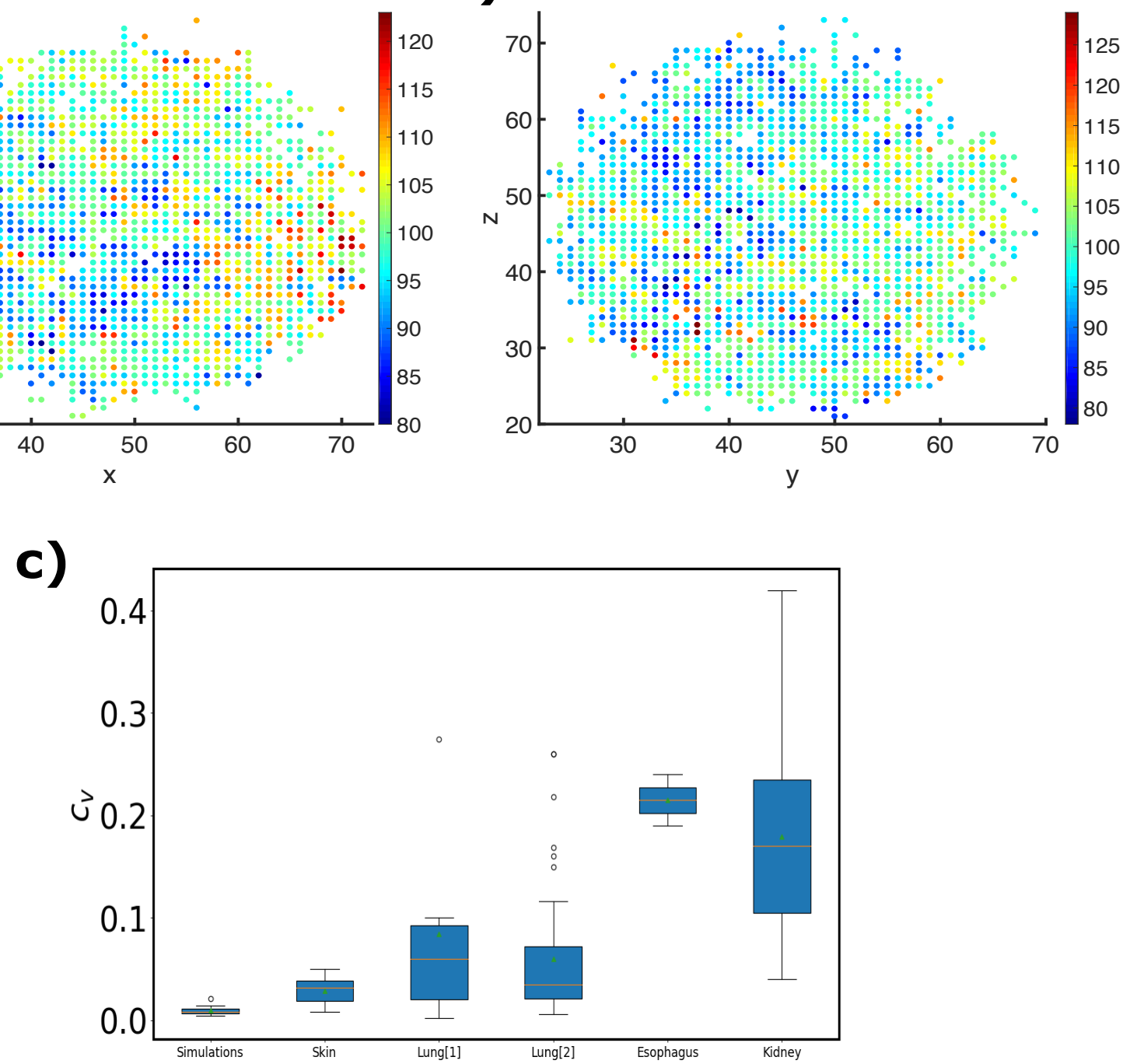

Figure 5: Spatial distribution of mutations (a), (b) $x-y$ and $y-z$ cross-section of the simulated 3D tumor. Distinct colors (see the color bar on the right) indicate the Tumor mutation burden (TMB) on each cell. Large variation in TMB occur throughout the simulated tumor. (c) Coefficient of variation, $c_{v}$, of TMB in simulation and the four cancer types. The $c_{v} \approx 0.008$, value in the simulations is small. For skin cancer dataset from Harbst et.al [42, the $c_{v}$ value is $\approx 0.023$. For lung cancer dataset from de Bruin et.al. [43, the $c_{v}$ value is $\approx 0.086$. The second Lung cancer dataset from Jamal et.al. 44, has an average $c_{v}$ of $\approx 0.066$. Esophageal cancer dataset from Cao et. al 45 has the highest $c_{v}$ value among the four cancer types $\left(c_{v} \approx 0.2\right)$. The kidney cancer dataset from Gerlinger et. al [20] has a high $c_{v}$ value of $\approx 0.178$. 

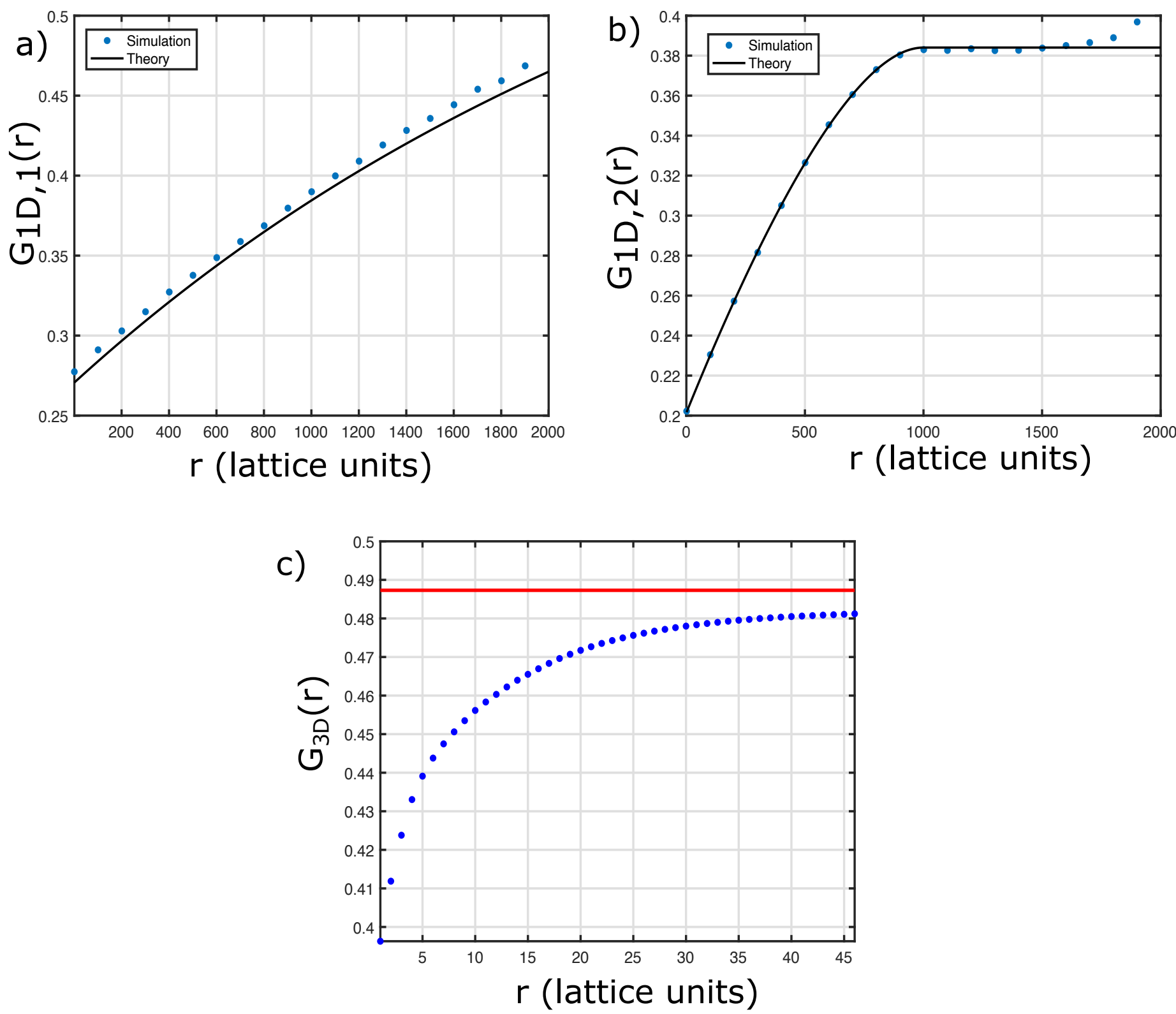

Figure 6: Spatial dependence of $\mathbf{G}(\mathbf{p})$ for different geometries. (a) Heterogeneity , $G_{1 D, 1}(r)(\mathrm{Eq} .12$ evaluated at $p=0.0005$ and $t=2,000)$, as a function of inter-cellular distance within a $1 \mathrm{D}$ semi-infinite lattice for $\alpha=1$. The black line is the result from theory (Eq, 12). The spatial dependence of $G_{1 D, 1}(r)$ arises because of the copying mechanism during cell-division, which introduces correlations between cells. (b) Heterogeneity, $G_{1 D, 2}(r)$ (Eq. 13 evaluated at $p=0.0003$ and $t=1,000)$ ), as a function of inter-cellular distance for a 1D infinite lattice. The blue dots correspond to simulations. (Continued on the next page) 
Figure 6: The black line is the theoretical result $(\mathrm{Eq}, 13)$. The curve saturates to the maximum allowed heterogeneity value given by equation $2 x_{t}\left(1-x_{t}\right)$. (c) Heterogeneity, $G_{3 D}(r)$ as a function of $r$ within a $3 \mathrm{D}$ tumor for $\alpha=1$. The dots in blue correspond to simulation for $p=0.02$. The tumor was evolved for 27 time steps, and had $\approx 50,000$ cells at the end of the simulations. The diameter of tumor is $\approx 46$ lattice units. The red line corresponds to $2 x_{t}\left(1-x_{t}\right)$ and is the maximum possible ITH value. 
bioRxiv preprint doi: https://doi org/10.1101/2022 02 19.481164 this version posted February 20,2022. The copyright holder for this

preprint (which was not certified by peer review) is the author/funder, who has granted bioRxiv a license to display the preprint in perpetuity. It is made available under aCC-BY-ND 4.0 International license.

a)

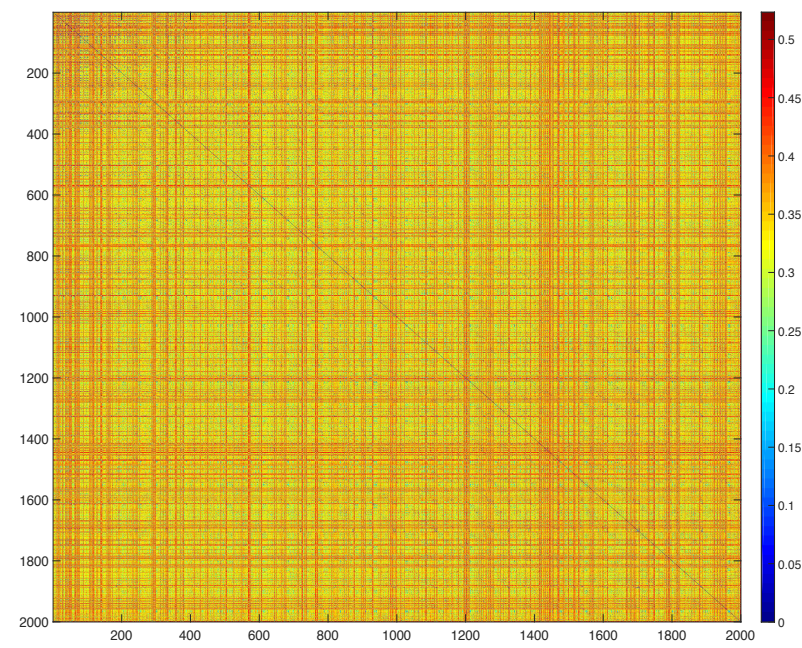

c)

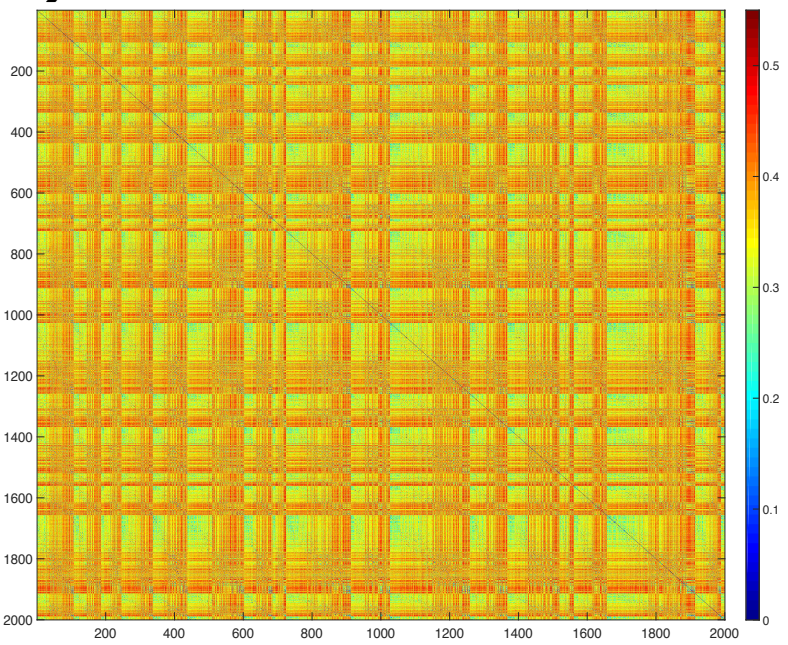

e)

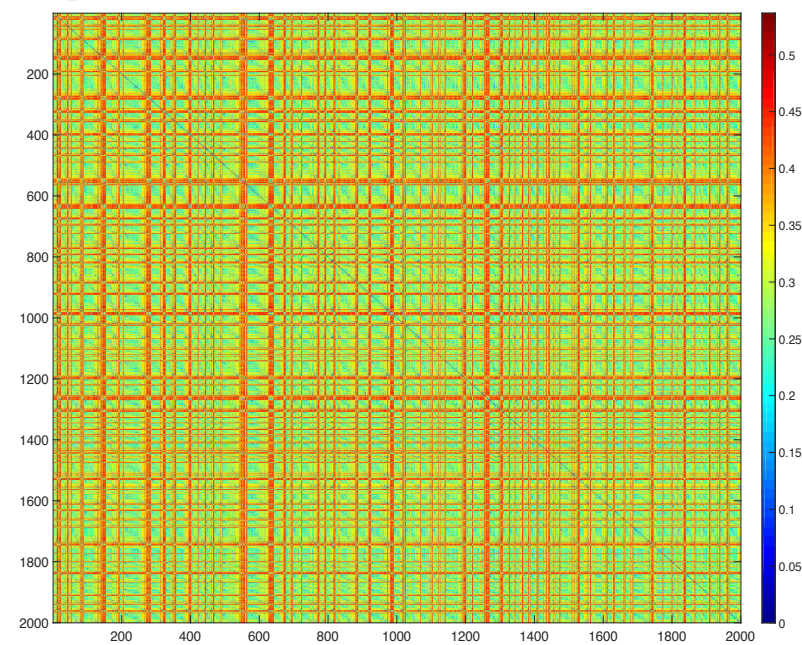

b)

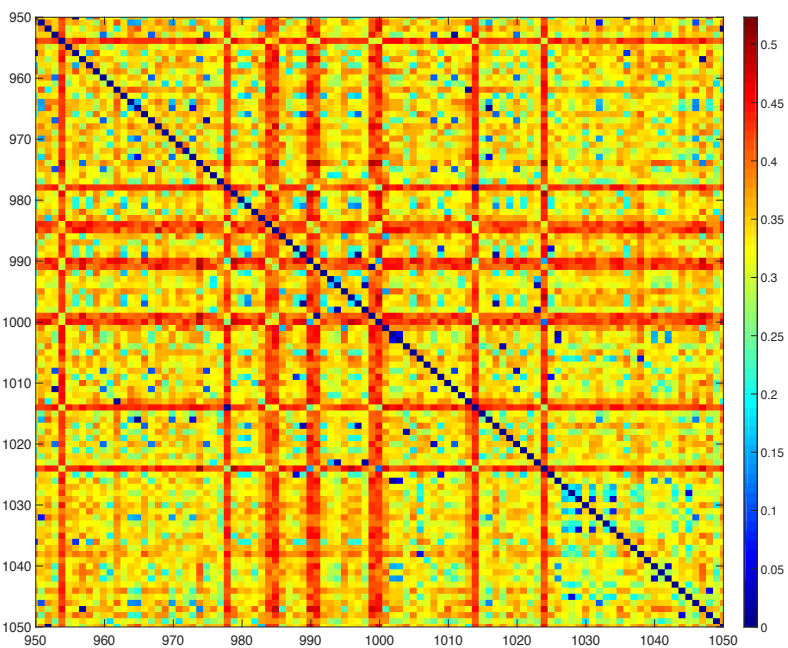

d)

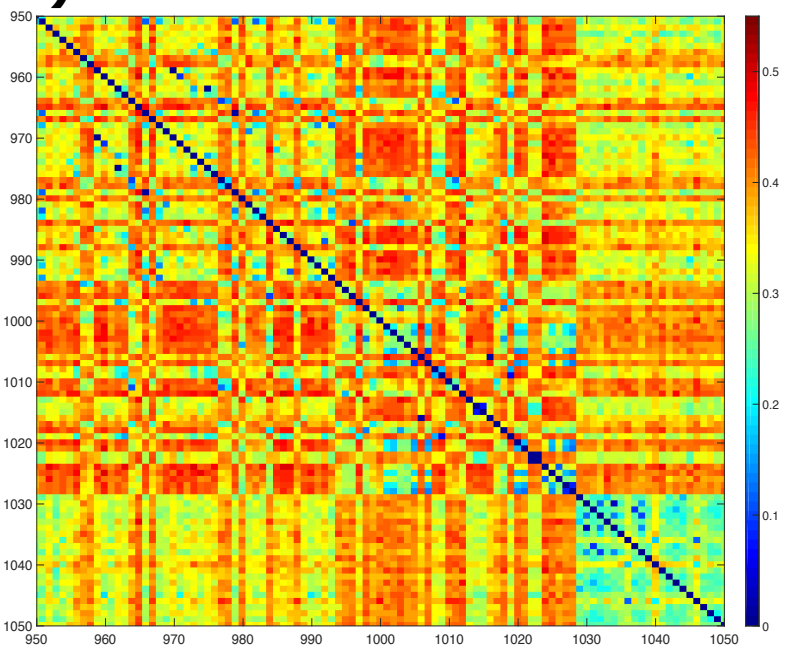

f)

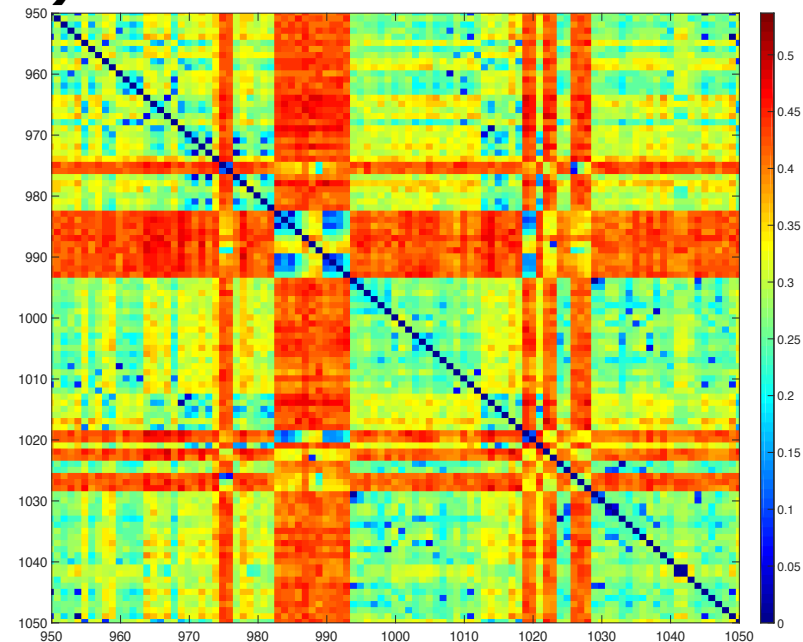

Figure 7: Hamming distance matrices for regions in the simulated tumor for $\alpha=0.55, p=0.003$ and $t=113$.(Continued on the next page) 
Figure 7: (a) Hamming matrix for 2,000 cells which are closest to the center of the simulated tumor. Each element $(i, j)$ of the matrix gives the values of the HD between a pair of cells. The color bar on the right corresponds to the HD values. (c) Same as (a) but for cells which are located approximately 20 lattice units away from the center of the tumor. (e) Same as (a) and (c) but for cells which are located approximately 27 lattice units away from the center of the tumor. (b, d, f) Zoomed in portion of size $100 \times 100$ from 950 to 1050 in Figures (a, c, e). The figures illustrate the sub-sample to sub-sample variations in the HD values depending on the location of the region sampled. The scales for HD are given on the right. 


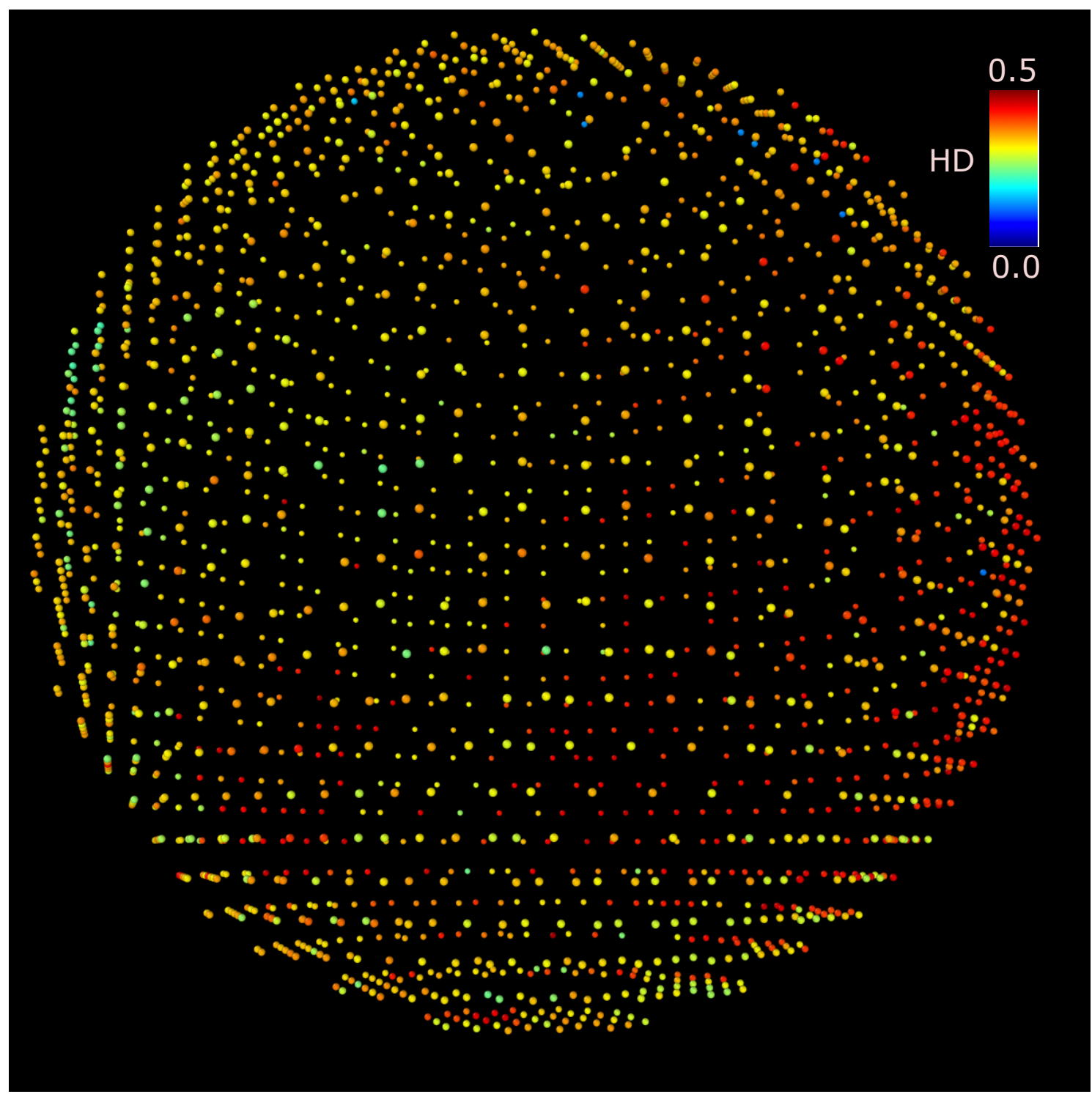

Figure 8: Visual depiction of the heterogeneity. The image was generated using 3D lattice simulations with $\alpha=0.55$ and $p=0.003$. The image corresponds to tumor evolution at $t=113$ generation. Figure shows the HD values for 2, 000 cells, depicted by their color, located approximately 15 lattice units from the center. The color bar, on the top right corner, shows the $H D$ scale. The large variation in the sub-population is evident. 
bioRxiv preprint doi: https://doi.org/10.1101/2022.02.19.481164; this version posted February 20, 2022. The copyright holder for this preprint (which was not certified by peer review) is the author/funder, who has granted bioRxiv a license to display the preprint in

[1] Berthier, L.; Biroli, G. Theoretical perspective on the glass transition and amorphous materials. Reviews of modern physics 2011, 83, 587.

[2] Kirkpatrick, T.; Thirumalai, D. Colloquium: Random first order transition theory concepts in biology and physics. Reviews of Modern Physics 2015, 8\%, 183.

[3] Thirumalai, D.; Mountain, R. D.; Kirkpatrick, T. Ergodic behavior in supercooled liquids and in glasses. Physical Review A 1989, 39, 3563.

[4] Altschuler, S. J.; Wu, L. F. Cellular heterogeneity: do differences make a difference? Cell 2010, 141, 559-563.

[5] Zahir, N.; Sun, R.; Gallahan, D.; Gatenby, R. A.; Curtis, C. Characterizing the ecological and evolutionary dynamics of cancer. Nature genetics 2020, 52, 759-767.

[6] Caswell-Jin, J. L.; Lorenz, C.; Curtis, C. Molecular Heterogeneity and Evolution in Breast Cancer. Annual Review of Cancer Biology 2021, 5, 79-94.

[7] McGranahan, N.; Swanton, C. Clonal heterogeneity and tumor evolution: past, present, and the future. Cell 2017, 168, 613-628.

[8] Heppner, G. H. Tumor heterogeneity. Cancer research 1984, 44, 2259-2265.

[9] Merlo, L. M.; Pepper, J. W.; Reid, B. J.; Maley, C. C. Cancer as an evolutionary and ecological process. Nature reviews cancer 2006, 6, 924-935.

[10] Tomasetti, C.; Li, L.; Vogelstein, B. Stem cell divisions, somatic mutations, cancer etiology, and cancer prevention. Science 2017, 355, 1330-1334.

[11] Tomasetti, C.; Vogelstein, B. Variation in cancer risk among tissues can be explained by the number of stem cell divisions. Science 2015, 34\%, 78-81.

[12] Manolio, T. A. Genomewide association studies and assessment of the risk of disease. New England journal of medicine 2010, 363, 166-176.

[13] Vogelstein, B.; Papadopoulos, N.; Velculescu, V. E.; Zhou, S.; Diaz, L. A.; Kinzler, K. W. Cancer genome landscapes. science 2013, 339, 1546-1558.

[14] Ciriello, G.; Magnani, L. The many faces of cancer evolution. Iscience 2021, 24, 102403

[15] Nowell, P. C. The clonal evolution of tumor cell populations. Science 1976, 194, 23-28.

[16] Marusyk, A.; Polyak, K. Tumor heterogeneity: causes and consequences. Biochimica et Biophysica Acta (BBA)-Reviews on Cancer 2010, 1805, 105-117.

[17] Hinohara, K.; Polyak, K. Intratumoral heterogeneity: more than just mutations. Trends in cell biology 2019, 29, 569-579.

[18] Michor, F.; Polyak, K. The origins and implications of intratumor heterogeneity. Cancer prevention research 2010, 19406207.

[19] Gerlinger, M.; Rowan, A. J.; Horswell, S.; Larkin, J.; Endesfelder, D.; Gronroos, E.; Martinez, P.; Matthews, N.; Stewart, A.; Tarpey, P., et al. Intratumor heterogeneity and branched evolution revealed by multiregion sequencing. New England journal of medicine 2012, 366, 883-892.

[20] Gerlinger, M.; Horswell, S.; Larkin, J.; Rowan, A. J.; Salm, M. P.; Varela, I.; Fisher, R.; McGranahan, N.; Matthews, N.; Santos, C. R., et al. Genomic architecture and evolution of clear cell renal cell carcinomas defined by multiregion sequencing. Nature genetics 2014, 46, 225.

[21] Swanton, C. Cancer therapeutics through an evolutionary lens. Journal of the Royal Society of Medicine 2018, $111,8-14$.

[22] Williams, M. J.; Werner, B.; Barnes, C. P.; Graham, T. A.; Sottoriva, A. Identification of neutral tumor evolution across cancer types. Nature genetics 2016, 48, 238-244.

[23] Sottoriva, A.; Kang, H.; Ma, Z.; Graham, T. A.; Salomon, M. P.; Zhao, J.; Marjoram, P.; Siegmund, K.; Press, M. F.; Shibata, D., et al. A Big Bang model of human colorectal tumor growth. Nature genetics 2015, 47, 209.

[24] Sun, R.; Hu, Z.; Curtis, C. Big bang tumor growth and clonal evolution. Cold Spring Harbor perspectives in medicine 2018, 8, a028381.

[25] Field, M. G.; Durante, M. A.; Anbunathan, H.; Cai, L. Z.; Decatur, C. L.; Bowcock, A. M.; Kurtenbach, S.; Harbour, J. W. Punctuated evolution of canonical genomic aberrations in uveal melanoma. Nature communications 2018, 9, 1-10.

[26] Davis, A.; Gao, R.; Navin, N. Tumor evolution: Linear, branching, neutral or punctuated? Biochimica et Biophysica Acta (BBA)-Reviews on Cancer 2017, 1867, 151-161.

[27] Li, X.; Thirumalai, D. Cooperation among tumor cell subpopulations leads to intratumor heterogeneity. Biophysical Reviews and Letters 2020, 15, 99-119.

[28] Burrell, R. A.; McGranahan, N.; Bartek, J.; Swanton, C. The causes and consequences of genetic heterogeneity in cancer evolution. Nature 2013, 501, 338-345.

[29] Swanton, C. Intratumor heterogeneity: evolution through space and time. Cancer research 2012 ,

[30] Morrissy, A. S.; Cavalli, F. M.; Remke, M.; Ramaswamy, V.; Shih, D. J.; Holgado, B. L.; Farooq, H.; Donovan, L. K.; Garzia, L.; Agnihotri, S., et al. Spatial heterogeneity in medulloblastoma. Nature genetics 2017, 49, 780.

[31] González-García, I.; Solé, R. V.; Costa, J. Metapopulation dynamics and spatial heterogeneity in cancer. Proceedings of the National Academy of Sciences 2002, 99, 13085-13089.

[32] Iwasa, Y.; Michor, F. Evolutionary dynamics of intratumor heterogeneity. PLoS One 2011, 6, e17866.

[33] Durrett, R.; Foo, J.; Leder, K.; Mayberry, J.; Michor, F. Intratumor heterogeneity in evolutionary models of tumor progression. Genetics 2011, genetics-110.

[34] Thalhauser, C. J.; Lowengrub, J. S.; Stupack, D.; Komarova, N. L. Selection in spatial stochastic models of cancer: 
bioRxiv preprint doi: https://doi.org/10.1101/2022.02.19.481164; this version posted February 20, 2022. The copyright holder for this preprint (which was not certified by peer review) is the author/funder, who has granted bioRxiv a license to display the preprint in perpetuity. It is made available under aCC-BY-ND 4.0 International license.

migration as a key modulator of fitness. Biology Direct 2010, 5, 21.

[35] Martens, E. A.; Kostadinov, R.; Maley, C. C.; Hallatschek, O. Spatial structure increases the waiting time for cancer. New journal of physics 2011, 13, 115014.

[36] Anderson, A. R.; Weaver, A. M.; Cummings, P. T.; Quaranta, V. Tumor morphology and phenotypic evolution driven by selective pressure from the microenvironment. Cell 2006, 127, 905-915.

[37] Waclaw, B.; Bozic, I.; Pittman, M. E.; Hruban, R. H.; Vogelstein, B.; Nowak, M. A. A spatial model predicts that dispersal and cell turnover limit intratumour heterogeneity. Nature 2015, 525, 261.

[38] Paterson, C.; Nowak, M. A.; Waclaw, B. An exactly solvable, spatial model of mutation accumulation in cancer. Scientific reports 2016, 6, 39511 .

[39] Antal, T.; Krapivsky, P.; Nowak, M. Spatial evolution of tumors with successive driver mutations. Physical Review E 2015, 92, 022705.

[40] Werner, B.; Case, J.; Williams, M. J.; Chkhaidze, K.; Temko, D.; Fernández-Mateos, J.; Cresswell, G. D.; Nichol, D.; Cross, W.; Spiteri, I., et al. Measuring single cell divisions in human tissues from multi-region sequencing data. Nature communications 2020, 11, 1-9.

[41] Sun, R.; Hu, Z.; Sottoriva, A.; Graham, T. A.; Harpak, A.; Ma, Z.; Fischer, J. M.; Shibata, D.; Curtis, C. Between-region genetic divergence reflects the mode and tempo of tumor evolution. Nature genetics 2017, 49, 1015-1024.

[42] Harbst, K.; Lauss, M.; Cirenajwis, H.; Isaksson, K.; Rosengren, F.; Torngren, T.; Kvist, A.; Johansson, M. C.; VallonChristersson, J.; Baldetorp, B., et al. Multi-region whole-exome sequencing uncovers the genetic evolution and mutational heterogeneity of early-stage metastatic melanoma. Cancer research 2016, canres-3476.

[43] de Bruin, E. C.; McGranahan, N.; Mitter, R.; Salm, M.; Wedge, D. C.; Yates, L.; Jamal-Hanjani, M.; Shafi, S.; Murugaesu, N.; Rowan, A. J., et al. Spatial and temporal diversity in genomic instability processes defines lung cancer evolution. Science 2014, 346, 251-256.

[44] Jamal-Hanjani, M.; Wilson, G. A.; McGranahan, N.; Birkbak, N. J.; Watkins, T. B.; Veeriah, S.; Shafi, S.; Johnson, D. H.; Mitter, R.; Rosenthal, R., et al. Tracking the evolution of non-small-cell lung cancer. New England Journal of Medicine 2017, 376, 2109-2121.

[45] Cao, W.; Wu, W.; Yan, M.; Tian, F.; Ma, C.; Zhang, Q.; Li, X.; Han, P.; Liu, Z.; Gu, J., et al. Multiple region wholeexome sequencing reveals dramatically evolving intratumor genomic heterogeneity in esophageal squamous cell carcinoma. Oncogenesis 2015, 4, e175.

[46] Zhang, J.; Fujimoto, J.; Zhang, J.; Wedge, D. C.; Song, X.; Zhang, J.; Seth, S.; Chow, C.-W.; Cao, Y.; Gumbs, C., et al. Intratumor heterogeneity in localized lung adenocarcinomas delineated by multiregion sequencing. Science 2014, 346, $256-259$

[47] Williams, M. J.; Sottoriva, A.; Graham, T. A. Measuring clonal evolution in cancer with genomics. Annual review of genomics and human genetics 2019, 20, 309-329.

[48] Zhai, W.; Lim, T. K.-H.; Zhang, T.; Phang, S.-T.; Tiang, Z.; Guan, P.; Ng, M.-H.; Lim, J. Q.; Yao, F.; Li, Z., et al. The spatial organization of intra-tumour heterogeneity and evolutionary trajectories of metastases in hepatocellular carcinoma. Nature communications 2017, 8, 4565.

[49] Jiang, T.; Shi, W.; Natowicz, R.; Ononye, S. N.; Wali, V. B.; Kluger, Y.; Pusztai, L.; Hatzis, C. Statistical measures of transcriptional diversity capture genomic heterogeneity of cancer. BMC genomics 2014, 15, 876 .

[50] Hormoz, S.; Desprat, N.; Shraiman, B. I. Inferring epigenetic dynamics from kin correlations. Proceedings of the National Academy of Sciences 2015, 112, E2281-E2289.

[51] Hormoz, S.; Singer, Z. S.; Linton, J. M.; Antebi, Y. E.; Shraiman, B. I.; Elowitz, M. B. Inferring cell-state transition dynamics from lineage trees and endpoint single-cell measurements. Cell systems 2016, 3, 419-433.

[52] El-Kebir, M.; Oesper, L.; Acheson-Field, H.; Raphael, B. J. Reconstruction of clonal trees and tumor composition from multi-sample sequencing data. Bioinformatics 2015, 31, i62-i70.

[53] Strachan, T.; Reid, A. Human Molecular Genetics; New York and London: Garland science, 1999.

[54] Grishkevich, V.; Yanai, I. Gene length and expression level shape genomic novelties. Genome research 2014, 24, $1497-1503$.

[55] Martincorena, I.; Raine, K. M.; Gerstung, M.; Dawson, K. J.; Haase, K.; Van Loo, P.; Davies, H.; Stratton, M. R.; Campbell, P. J. Universal patterns of selection in cancer and somatic tissues. Cell 2017, 171, 1029-1041.

[56] Li, X.; Thirumalai, D. Imprints of tumor mutation burden on chromosomes and relation to cancer risk in humans: A pan-cancer analysis. bioRxiv 2020.04.20.050989 2021, 2020-04.

[57] Tarabichi, M.; Martincorena, I.; Gerstung, M.; Leroi, A. M.; Markowetz, F.; Spellman, P. T.; Morris, Q. D.; Lingjærde, O. C.; Wedge, D. C.; Van Loo, P. Neutral tumor evolution? Nature genetics 2018, 50, 1630-1633.

[58] Cannataro, V. L.; Townsend, J. P. Neutral theory and the somatic evolution of cancer. Molecular biology and evolution 2018, 35, 1308-1315.

[59] McDonald, T. O.; Chakrabarti, S.; Michor, F. Currently available bulk sequencing data do not necessarily support a model of neutral tumor evolution. Nature genetics 2018, 50, 1620-1623.

[60] Tung, H.-R.; Durrett, R. Signatures of neutral evolution in exponentially growing tumors: A theoretical perspective. PLOS Computational Biology 2021, 17, e1008701.

[61] Wang, H.-Y.; Chen, Y.; Tong, D.; Ling, S.; Hu, Z.; Tao, Y.; Lu, X.; Wu, C.-I. Is the evolution in tumors Darwinian or non-Darwinian? National Science Review 2018, 5, 15-17.

[62] Navin, N.; Kendall, J.; Troge, J.; Andrews, P.; Rodgers, L.; McIndoo, J.; Cook, K.; Stepansky, A.; Levy, D.; Esposito, D., et al. Tumour evolution inferred by single-cell sequencing. Nature 2011, 472, 90.

[63] Meacham, C. E.; Morrison, S. J. Tumour heterogeneity and cancer cell plasticity. Nature 2013, 501, 328-337.

[64] Li, X.; Thirumalai, D. A mathematical model for phenotypic heterogeneity in breast cancer with implications for therapeutic 
strategies. bioRxiv 2021.06.04.447174 2021,

[65] Moscow, J. A.; Fojo, T.; Schilsky, R. L. The evidence framework for precision cancer medicine. Nature Reviews Clinical Oncology 2018, 15, 183-192.

[66] Valencia, A. M. J.; Wu, P.-H.; Yogurtcu, O. N.; Rao, P.; DiGiacomo, J.; Godet, I.; He, L.; Lee, M.-H.; Gilkes, D.; Sun, S. X., et al. Collective cancer cell invasion induced by coordinated contractile stresses. Oncotarget 2015, 6, 43438.

[67] Han, Y. L.; Pegoraro, A. F.; Li, H.; Li, K.; Yuan, Y.; Xu, G.; Gu, Z.; Sun, J.; Hao, Y.; Gupta, S. K., et al. Cell swelling, softening and invasion in a three-dimensional breast cancer model. Nature physics 2020, 16, 101-108.

[68] Martino, N.; Kwok, S. J.; Liapis, A. C.; Forward, S.; Jang, H.; Kim, H.-M.; Wu, S. J.; Wu, J.; Dannenberg, P. H.; Jang, S.-J., et al. Wavelength-encoded laser particles for massively multiplexed cell tagging. Nature photonics 2019, 13, $720-727$.

[69] Malmi-Kakkada, A. N.; Li, X.; Samanta, H. S.; Sinha, S.; Thirumalai, D. Cell Growth Rate Dictates the Onset of Glass to Fluidlike Transition and Long Time Superdiffusion in an Evolving Cell Colony. Physical Review X 2018 , 8, 021025.

[70] Sinha, S.; Thirumalai, D. Self-generated persistent random forces drive phase separation in growing tumors. The Journal of Chemical Physics 2020, 153, 201101.

[71] Sinha, S.; Malmi-Kakkada, A. N.; Li, X.; Samanta, H. S.; Thirumalai, D. Spatially heterogeneous dynamics of cells in a growing tumor spheroid: Comparison between theory and experiments. Soft Matter 2020, 16, 5294-5304.

[72] Samanta, H. S.; Sinha, S.; Thirumalai, D. Far from equilibrium dynamics of tracer particles embedded in a growing multicellular spheroid. arXiv:2003.12941 2020,

[73] Sinha, S.; Malmi-Kakkada, A. N. Inter-particle adhesion regulates the surface roughness of growing dense three-dimensional active particle aggregates. Journal of Physical Chemistry B 2021, 125, 10445-10451.

[74] Malmi-Kakkada, A. N.; Sinha, S.; Li, X.; Thirumalai, D. Adhesion strength between cells regulate non-monotonic growth by a biomechanical feedback mechanism. bioRxiv 2021,

[75] Sinha, S.; Li, X.; Das, R.; Thirumalai, D. Mechanical feedback controls the emergence of dynamical memory in growing tissue monolayers. arXiv:2202.04806 2022, 